\title{
An atlas of line profile studies for SU UMa type cataclysmic variables ${ }^{\star} \star \star$
}

\author{
C. Tappert ${ }^{1}$, R. E. Mennickent ${ }^{1}$, J. Arenas ${ }^{1}$, K. Matsumoto ${ }^{2, \star \star \star}$, and R. W. Hanuschik ${ }^{3}$ \\ ${ }^{1}$ Universidad de Concepción, Departamento de Física, Casilla 160-C, Concepción, Chile \\ 2 Graduate School of Natural Science and Technology, Okayama University, Okayama 700-8530, Japan \\ ${ }^{3}$ ESO, Karl-Schwarzschild-Str. 2, 85748 Garching, Germany
}

Received 8 April 2002 / Accepted 12 June 2003

\begin{abstract}
We present $\mathrm{H} \alpha$ line-profile analyses for the seven SU UMa type dwarf novae AK Cnc, WX Cet, AQ Eri, VW Hyi, RZ Leo, TU Men, and HS Vir. All data sets are treated in the same manner, applying a sequence of techniques for each system. The basic ingredients of this sequence are the diagnostic diagram to determine the zero point of the orbital phase, and Doppler tomography to visualise the emission distribution. We furthermore introduce a new qualitative way of to evaluate the Doppler fit, by comparing the line profile of the reconstructed with the original spectrum in the form of the $V / R$ plot. We present the results of the analysis in the compact form of an atlas, allowing a direct comparison of the emission distribution in our targets. Although most of the data sets were not taken with the intention of a line-profile analysis, we obtain significant results and are able to indicate the type of the additional emission in these systems. Our objects should have in principle very similar physical properties, i.e. they cover only a small range in orbital periods, mass ratios, and mass-transfer rates. Nevertheless, we find a large variety of phenomena both with respect to the individual systems and also within individual data sets of the same object. This includes "canonical" additional emission components from the secondary star and the bright spot, but also emission from the leading side of the accretion disc.
\end{abstract}

Key words. accretion, accretion disks - line: profiles - methods: data analysis - astronomical data bases: miscellaneous novae, cataclysmic variables

\section{Introduction}

Cataclysmic variables (CVs) are close interacting binaries with a white dwarf as primary and a late-type main-sequence star as secondary component. The latter fills its critical Roche lobe and thus enables mass transfer through the inner Lagrangian point into the Roche volume of the primary. In the absence of strong magnetic fields, the gas stream from the secondary star dissipates and forms an accretion disc around the white dwarf over which the mass transfer takes place.

The accretion disc is the dominant light source in these systems. It is also the primary origin of the strong emission lines especially of the $\mathrm{H}$ and $\mathrm{He}$ series, which are characteristic for a CV spectrum (e.g. Smak 1981; Horne \& Marsh 1986; Horne 1995). In most CVs, the disc is too bright to allow the detection of the absorption features of the primary and the secondary. The emission lines thus represent the major source of information on the orbital motion. Furthermore, the assumption of a Keplerian velocity law allows the derivation of several system

Send offprint requests to: $\mathrm{C}$. Tappert, e-mail: claus@gemini.cfm.udec.cl

* Partly based on observations at ESO telescopes.

$\star \star$ Figures A.1, A.2, and A.7 to A.21 are only available in electronic form at http://www. edpsciences.org

$\star \star \star$ Present address: Astronomical Institute, Osaka Kyoiku University, Asahigaoka 4-698-1, Kashiwara, Osaka 582-8582, Japan. parameters like masses and inclination. These parameters are, of course, vital for an understanding of the physics in these systems, and furthermore represent the only means to test theoretical models on CV evolution (see, e.g. Warner 1995 for a comprehensive overview on $\mathrm{CVs}$ ).

This approach is only feasible if the emission lines originate in a region moving with the same mean velocity vector as the primary star. Only in this case do they track its orbital motion. However, several examples show that, additionally to the disc-borne emission, other emission components distort the originally symmetric line profile (e.g. Stover 1981). The most prominent sources of this emission are the region around the bright spot (the place where the gas stream impacts on the disc), the gas stream from the secondary star, or an irradiated part on the surface of the latter, but also apparently enhanced emission regions in the accretion disc, which, in a number of cases, make a physical explanation difficult (Tappert 1999; North et al. 2001). All these regions have in common that they are "isolated" in the sense that they are not centred symmetrically on the white dwarf. Consequently, they have both different velocity amplitudes and phasing with respect to the primary. The radial velocity parameters of the combined line profile as a whole thus will not reflect the motion of the white dwarf, leading to erroneous system parameters. We will call these asymmetric emission regions in the following "isolated emission sources" (IES). 
Fortunately, in most cases, the distorting components are confined to the line centre ${ }^{1}$. A careful examination of the line profile is thus usually able to separate the influences of additional components and allows the determination of the system parameters with some confidence. Furthermore, a recent statistical study showed that the additional components represent more than just a mere obstacle in the derivation of the system parameters, but in principle provide valuable information on the physical processes in CVs (Tappert \& Hanuschik 2001). Last, not least, the discovery of spiral shocks in CV discs by the means of Doppler tomography (Steeghs et al. 1997) proves the importance of line profile analyses for the understanding of accretion physics.

Kaitchuck et al. (1994) were the first to re-analyse a large sample of "old", i.e. already elsewhere published, CV spectra with a newly developed technique. Their atlas of Doppler maps allowed a direct comparison of the various types of emission distribution. The atlas included 18 systems, 3 of them with an orbital period shorter than 3 hours.

In a similar spirit, the scope of the present paper is to apply a sequence of techniques of line profile analysis to a sample of CVs. Our concentration on short-period dwarf novae allows us to study the variety of phenomena presented by systems which can be assumed to have very similar physical properties, i.e. a low mass ratio $q=M_{2} / M_{1} \leq 0.3$ and low mass-transfer rates of the order $\dot{M} \sim 10^{-11} M_{\odot} /$ yr. Furthermore, according to standard CV evolution theory, low mass transfer rates imply optically thin discs. These are strong line emitters and could reveal structural details more easily than optically thick discs.

The present atlas also provides a significant increase of Doppler maps for short-period CVs, whose number of available line-profile analyses is in strong mismatch with respect to the number of known systems, compared with long-period CVs (Tappert \& Hanuschik 2001). This is basically due to the fact that for these faint systems the orbital period is easily accessible for small telescopes through the superhump phenomenon, while detailed studies need large telescopes. The latter therefore usually concentrate on a few "interesting" objects, like WZ Sge (e.g., Skidmore et al. 2000; and references therein). Our present sample instead is not part of this "selection effect", as none of the systems has (yet) been the subject of a more detailed investigation involving the necessary large telescopes. It therefore adds to a more "unbiased" view of the properties of SU UMa stars.

Following our goal of direct comparison, we restrict our analysis to the $\mathrm{H} \alpha$ profile, which also represents the most accessible emission line.

\section{The sample}

The systems in question are the seven SU UMa type dwarf novae AK Cnc, WX Cet, AQ Eri, VW Hyi, RZ Leo, TU Men, and HS Vir. With the exception of VW Hyi, the data were not taken with the intention to perform a line-profile analysis, but to determine the orbital period. The observational background

\footnotetext{
1 The high velocity wings in SW Sex systems are an important exception (e.g. Hoard et al. 2000, and references therein).
}

and the properties of the data have been published elsewhere, together with the results of the initial analysis. In Table 1 we therefore present only a summary of the basic parameters of the data sets, and refer the reader to the respective publications listed in the table for further details.

The available data on HS Vir actually consists of more than the one set covered here. However, the system showed large, apparently erratic, variations from one set to another, and also within individual data sets. A more detailed analysis is required in this case, which is beyond the scope of the present paper, since it aims at a uniform treatment of the data. We have therefore included only the "most well behaved" data set of this object as an example.

\section{Analysis sequence}

The following sequence of analysis techniques is applied to each data set:

1. Visual inspection: apart from the changes due to the orbital motion and/or to additional emission sources, the emission-line profile is also affected by luminosity changes of the system. Roughly, the strength of the disc-borne emission lines is expected to decrease with increasing disc brightness, due to the increasing optical thickness of the accretion disc. Additional emission components, however, might be affected quite differently by such a variation, so that their influence becomes stronger (e.g., emission from the secondary star) or weaker (e.g., emission from the bright spot). Line profiles of the same $\mathrm{CV}$ in different states thus usually show quite different phenomena (compare, e.g., Martínez-Pais et al. 1994, 1996, for SS Cyg in quiescence and outburst, respectively). A visual comparison of line profiles of different data sets therefore provides a first hint as to whether a combination of these data is possible in order to increase the phase resolution, or if each set has to be treated individually.

Similarly, in high inclination systems, the brightness variation due to the orbital motion can affect the line profile at specific orbital phases. This is especially true for eclipsing systems, where also the line emission source is partly or completely obscured (e.g. Kaitchuck et al. 1998). These parts of the data sets usually have to be excluded from the line profile analysis.

2. Diagnostic diagram: this method makes use of the doubleGaussian convolution with the line profile (Schneider \& Young 1980; Shafter 1983b), which allows to measure radial velocities of different parts of the line. These velocities $v_{\mathrm{r}}(\varphi)$ can be fitted with a sinusoidal function

$v_{\mathrm{r}}(\varphi)=\gamma-K_{\mathrm{em}} \sin \left(2 \pi\left(\varphi-\varphi_{0}\right)\right)$,

to yield the systemic velocity $\gamma$, the semi-amplitude $K_{\mathrm{em}}$, and the fiducial phase corresponding to superior conjunction of the primary star $\varphi_{0}$. When these parameters are plotted as a function of the separation $d$ of the two Gaussians, a so-called diagnostic diagram is obtained, which illustrates the variation of the parameters as different parts of the line are measured (Shafter 1983a). In order to evaluate the significance of 
the resulting variation, the corresponding error of the semiamplitude $\sigma\left(K_{\mathrm{em}}\right) / K_{\mathrm{em}}$ is included in the plot. A sharp increase of this value at large separations indicates the point beyond which the noise begins to dominate the signal from the line wings and the parameters become unreliable. The values belonging to the largest separation $d_{\max }$ before this happens are usually chosen as the best possible approximation to the correct ones, so that $K_{\mathrm{em}}=K_{1}$, i.e. the true semi-amplitude of the orbital motion of the primary.

A second criterion is based on the expectation that the parameters should (again) approach constant values at large separations when additional emission sources cease to influence the line profile. This is usually the stronger criterion, as the first one is often ambiguous.

The diagnostic diagram furthermore allows us to fix the fiducial phase, which is a prerequisite for the evaluation of the subsequent Doppler mapping.

3. Doppler tomography: this technique, introduced by Marsh $\&$ Horne (1988) to the realm of interacting binaries, interprets the one-dimensional snapshots represented by a spectrum taken at a specific orbital phase as projections of a two-dimensional emission distribution. The latter is reconstructed as a map in velocity space via an inversion of these projections (see Marsh 2001 for a recent review).

In this work, the implementation of Spruit (1998) has been used. We have replaced the original IDL routines by a corresponding MIDAS interface, but still use the FORTRAN core program (version 2.3.1), to run the computation on a Linux PC. The input spectra have first been continuum subtracted, the extracted line profiles have then been normalised in flux (in order to avoid too small numbers for the Doppler routine, the total line flux has been set to 100) to minimise artefacts due to intensity variations. A smearing kernel of 2 pixels was used to compute the Doppler maps, which were subsequently smoothed with a $3 \times 3$ pixel $(11 \times 11$ in the case of VW Hyi) averaging filter for noise reduction. Tests with larger kernels showed that the visual information content of the resulting map basically remains the same, but that the profile comparison (see step 4) suffered from too strong smoothing. A kernel size of 1 pixel, on the other hand, in almost all cases led to infinite iteration loops due to the noise becoming dominant. All other input parameters for the Doppler routine were set to default values (Spruit 1998).

The $\gamma$ velocity resulting from the diagnostic diagram was taken as a first guess for correcting the input line to its rest wavelength. It was then iteratively adjusted by comparing the reconstructed and original average spectra.

The resulting Doppler maps are orientated as usual, i.e. the fiducial phase is at $v_{x}=0$ and $v_{y}>0$ and increases in clockwise direction. If the phase corresponds to the orbital motion this results in the white dwarf being located at $\left(v_{x}, v_{y}\right)=\left(0,-K_{1}\right)$ and the secondary star at $\left(0, K_{2}\right)$.

4. V/R-plot: perhaps the simplest way to examine a line profile for asymmetry is to compare its blue part to its red one. This has been done in the past for double-peaked profiles by computing the ratio between the intensity of the blue (violet) peak and the one of the red peak, the so-called $V / R$ ratio (e.g., Mennickent 1994). In order to apply this method also to singlepeaked profiles, one can define a different, but equivalent, $V / R$ as (Tappert 1999)

$$
V / R=\log \frac{F(V)}{F(R)}
$$

where $F(V)$ is not the peak intensity but the flux of the blue part and $F(R)$ the corresponding one of the red part. The point which separates both halves, $\lambda_{c}$, is chosen as the centre of the line flanks at a specific intensity value which has to be low enough to avoid being affected by the central valley in doublepeaked profiles, and high enough to avoid the noise-dominated extreme line wings. The sigma of the individual $V / R$ value is computed by a Monte Carlo simulation which adds a random value, uniformly distributed within an interval determined by the $\mathrm{S} / \mathrm{N}$, to each data point in the spectrum, and measures the $V / R$ for a thousand of such newly computed data sets.

We here use the $V / R$ plot to compare the original spectrum with the reconstructed one from the Doppler map. In this way it can be quantitatively evaluated if the Doppler tomography has been able to reproduce the general shape of the individual line profiles.

In the atlas presented in Appendix A, we furthermore include two-dimensional greyscale plots of the original and the difference data, the latter being computed by subtracting the reconstructed spectrum from the original one. In this way, also possible intensity deficits (black features in the plot) or excesses (white features) of the reconstructed data become visually clear.

\section{Results}

\subsection{Splitting of data sets}

For all objects, the analysis methods were first conducted on each individual data set (i.e., corresponding to one night of observation; see Table 1). Where no significant differences were found, the analysis was repeated on the combined set in order to minimise noise.

The data of AQ Eri represent a special case. Here, the line profiles proved to vary both in strength and in shape on nonorbital timescales (compare especially phases 0.1 and 0.8 in the upper plot of Fig. A.7). We therefore split the set in three parts 1a, 1b, 1c, with each set covering one full orbit. Set $1 \mathrm{~b}$ overlaps with the other two (it includes 4 spectra from set 1a and 3 from set $1 \mathrm{c}$ ), but sets $1 \mathrm{a}$ and $1 \mathrm{c}$ are independent of each other.

The appearance of the line profiles of HS Vir (upper plot of Fig. A.20) suggests a similar treatment. However, here the phase distribution of the data did not allow a division into independent sets which would cover a complete orbit. We conducted the analyses for different subsets, but did not find any significant differences with respect to results or residuals. 
Table 1. General properties of the data. The dates of observations and the corresponding data sets (numbers usually refer to individual nights; for exceptions see text) are presented in Cols. 2 and 3, respectively. Column 4 gives the total number of spectra available for the analysis, Col. 5 their spectral resolution $(F W H M)$, Col. 6 the telescope used for the observations, and the last column lists references concerning the previous publication of the data sets.

\begin{tabular}{lllllll}
\hline \hline star & dates & sets & $n_{\mathrm{sp}}$ & $\Delta \lambda[\AA]$ & telescope & reference \\
\hline AK Cnc & $1995-03-19,21,22$ & $1-3$ & 40 & 4.5 & $2.5 \mathrm{~m} \mathrm{LCO}$ & Arenas \& Mennickent (1998) \\
WX Cet & $1992-12-07,08,10$ & $1-3$ & 37 & 2.0 & $2.2 \mathrm{~m}$ ESO/MPI & Mennickent (1994) \\
AQ Eri & $1992-12-11$ & $1(\mathrm{a}-\mathrm{c})$ & 44 & 2.0 & $2.2 \mathrm{~m} \mathrm{ESO/MPI}$ & Mennickent (1995b) \\
VW Hyi & $1994-10-15$ to 17 & $1-3$ & 64 & 1.1 & $1.9 \mathrm{~m} \mathrm{SAAO}$ & Tappert (1999) \\
RZ Leo & $1995-02-10,11,03-21,23$ & $1 \mathrm{a}, 1 \mathrm{~b}, 2 \mathrm{a}, 2 \mathrm{~b}$ & 46 & $6.0,4.5$ & $2.2 \mathrm{~m} \mathrm{ESO/MPI,} \mathrm{2.5} \mathrm{m} \mathrm{LCO}$ & Mennickent \& Tappert (2001) \\
TU Men & $1992-12-09,10,12,14$ & $1-4$ & 67 & 2.0 & $2.2 \mathrm{~m} \mathrm{ESO/MPI}$ & Mennickent (1995a) \\
HS Vir & $1998-05-30$ & 1 & 34 & 2.5 & $3.5 \mathrm{~m} \mathrm{NTT}$ & Mennickent et al. (1999) \\
\hline
\end{tabular}

\subsection{Diagnostic diagrams}

The diagnostic diagrams proved to be robust with respect to the differences between the individual data sets. With the exception of RZ Leo, where the insufficient precision of the orbital period does not allow for an interpolation of the phases, we therefore used the combined data sets.

In the present application of the diagnostic diagram we are mostly interested in determining the zero point of the orbital phase, on which depends the interpretation of the subsequent Doppler maps. For the majority of the objects in our sample, this choice was unambiguous, as after clearing distortions due to structure in the line core this parameter remained constant over a large range of Gaussian separations. We therefore based our choice of the maximum separation $d_{\max }$ on the stability of the other parameters (of $\gamma$ in the case of AK Cnc and WX Cet, $\gamma$ and $K_{\mathrm{em}}$ for TU Men) where the noise parameter $\sigma\left(K_{\mathrm{em}}\right) / K_{\mathrm{em}}$ appeared not to be reliable, on a combination of the noise and the constancy criteria (AQ Eri), or (for HS Vir) only on the noise behaviour. In the cases of VW Hyi and RZ Leo, $\varphi_{0}$ does not reach constancy within the allowed range with respect to the noise criterion. We here chose the values corresponding to the largest separation within this range. Judging from the appearance of the variation of $\varphi_{0}$ at the separations before this point (slight monotonic decline), we do not expect the error in the determination of the fiducial phase to be larger than $\sim 0.1$ orbits.

For completeness, we have listed the parameters corresponding to our choice of $d_{\max }$ in Table 2.

\subsection{Doppler maps}

The general appearance of the Doppler maps is a rather noisy one. This is due to the fact that the $\mathrm{S} / \mathrm{N}$ of the data could not be improved in the majority of our systems by co-adding phases of different orbits because of the observed short- and long-term variations. For the same reason also the phase coverage often stays incomplete and/or non-uniform. All this gives rise to artefacts in the Doppler maps, and one has therefore to be careful not to over-interpret certain features.

Nevertheless, the tests by the means of the trailed spectrograms and the $V / R$ plots show that the reconstructed spectra produced by the Doppler fitting agree fairly well with the
Table 2. Radial-velocity parameters for $\mathrm{H} \alpha$ as derived by the analysis of the diagnostic diagram. Column 2 gives the FWHM of the Gaussians used for the diagram, Col. 3 the critical separation, Cols. 4 and 5 contain the resulting semi-amplitude and the error of the zero phase, respectively. The $\gamma$ parameter is not listed, as it reflects more the wavelength calibration process than a real physical parameter.

\begin{tabular}{lclcll}
\hline \hline data set & $\begin{array}{c}F W H M \\
{[\AA]}\end{array}$ & $\begin{array}{l}d_{\max } \\
{[\AA]}\end{array}$ & $\begin{array}{c}K_{\mathrm{em}} \\
{[\mathrm{km} / \mathrm{s}]}\end{array}$ & $\begin{array}{l}\sigma\left(\varphi_{0}\right) \\
{[\text { orbits] }}\end{array}$ & Fig. \\
\hline AK Cnc & 4 & 34 & $42(09)$ & 0.036 & A.1 \\
WX Cet & 4 & 52 & $59(18)$ & 0.061 & A.3 \\
AQ Eri & 2 & 44 & $36(09)$ & 0.042 & A.7 \\
VW Hyi & 4 & 40 & $38(09)$ & 0.039 & A.11 \\
RZ Leo 1 & 4 & 66 & $49(13)$ & 0.042 & - \\
RZ Leo 2 & 4 & 62 & $51(15)$ & 0.050 & A.15 \\
TU Men & 2 & 48 & $84(09)$ & 0.017 & A.18 \\
HS Vir & 2 & 64 & $98(09)$ & 0.017 & A.20 \\
\hline
\end{tabular}

original data, with the exception of HS Vir (see Sect. 4.3.7). Differences in the $V / R$ values (e.g., lower plots for WX Cet, Figs. A.4 and A.6; RZ Leo, Fig. A.17) can largely be attributed to noise in the original spectra and to the fact that due to the Doppler smoothing (or smearing) the $\mathrm{S} / \mathrm{N}$ in the reconstructed spectra is often up to 5 times higher. We therefore conclude that the line profiles themselves have been well reproduced. As shown by the trailed spectrograms, in general the same can be said for the intensity behaviour, with a few notable exceptions which are discussed below for the respective systems.

\subsubsection{AK Cnc}

The Doppler map shows a symmetric emission distribution (Fig. A.2). AK Cnc therefore appears to be one of the few systems which show no presence of any IES; the survey of Tappert \& Hanuschik (2001) found only 4 such objects in a sample of $68 \mathrm{CVs}$ (not including AK Cnc). Unfortunately, the system was not observed in quiescence, but in decline from outburst. It thus remains unclear if the absence of IES is caused by a still optically thick disc (i.e. if this is only a temporary absence), or simply due to the low inclination of the system.

\subsubsection{WX Cet}

The Doppler maps for the WX Cet are amongst those with the most noisy appearance (Figs. A.4-A.6). The low S/N of the 


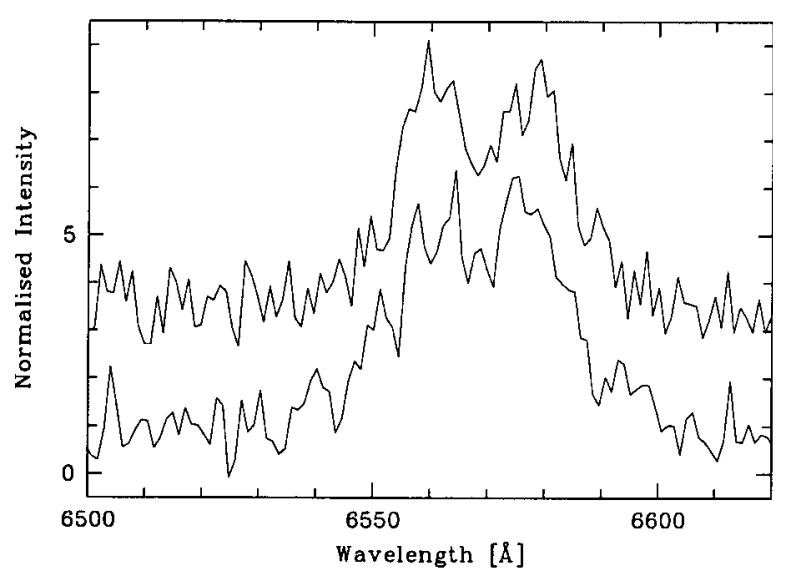

Fig. 1. Example of an original continuum-normalised spectrum of the WX Cet data set (bottom) and its corresponding model spectrum (top). The latter has been displaced vertically by 2.5 intensity units.

data is probably also responsible for the fact that the clear signature of disc emission, i.e. a ring-shaped emission distribution centred on the primary, is not seen, although the line profiles are double-peaked (Fig. A.3). Instead all maps show a rather spotty emission distribution, however, with a maximum on the leading side (i.e. $v_{x}>0$ ) as a common feature.

The low $\mathrm{S} / \mathrm{N}$ and the non-uniform phase distribution make the WX Cet data sets the ideal test case to examine the significance of the features in the Doppler map. For this, we established a set of model spectra using the line profile prescription by Smak (1981). We adjusted the model parameters until a visually good agreement with the shape and quality of the original WX Cet data was reached. The model data were displaced in wavelength according to Eq. (1), using the phases of the WX Cet data and the parameters obtained by the application of the diagnostic diagram $\left(K_{1}=59 \mathrm{~km} \mathrm{~s}^{-1}\right)$ and the Doppler tomography $\left(\gamma=260 \mathrm{~km} \mathrm{~s}^{-1}\right)$. Figure 1 gives an example of the resulting model spectrum.

These spectra represent a symmetric disc emission, without any additional component. The corresponding Doppler maps (Fig. 2), however, show the same spotty appearance as for the original data set, with the ring-shaped disc signature being only slightly more evident. The low intensity arcs and blobs in many of the Doppler maps (see also the VW Hyi data, especially Fig. A.13) can also be explained by the data quality. It even appears not improbable that the individual maps of WX Cet could be simply reproduced by noisy spectra without the need to invoke an additional component (compare especially the map for model set 2 with the WX Cet data). However, the fact that all WX Cet maps show an emission maximum on the leading side of the disc indicates that this represents a real feature.

\subsection{3. $A Q$ Eri}

The maps for the individual data sets show significant differences (Figs. A.8-A.10), with an emission maximum close to the possible position of the secondary star at $\left(0, K_{2}\right)$ moving to a position in the lower left quadrant of the Doppler map. However, the important difference to the behaviour in

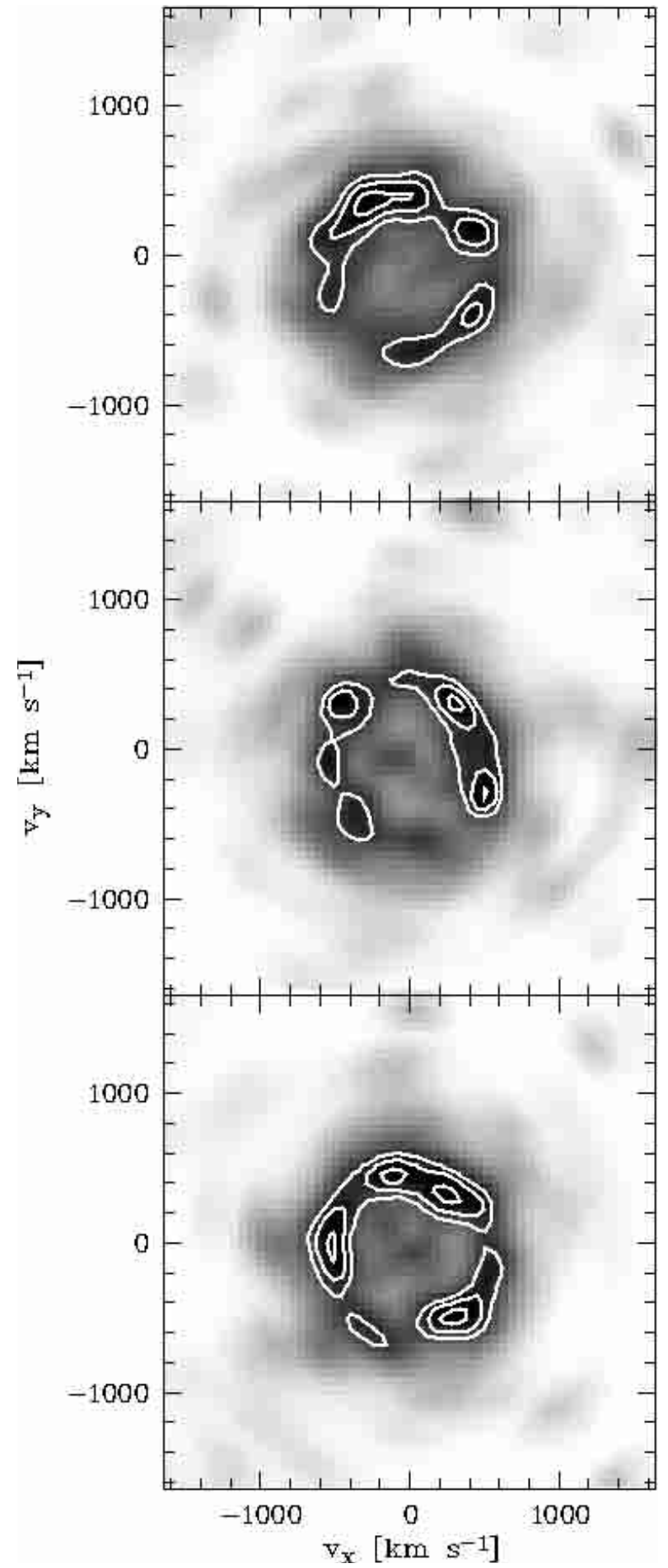

Fig. 2. Doppler maps for the model spectra with respect to the WX Cet data sets 1-3 (top to bottom).

other systems (e.g. VW Hyi) is that this evolution takes place over $\sim 2.5$ orbits, i.e. on very short timescales. It is therefore doubtful if the maps in this case are representative of the emission distribution at all, or if the timescales of the profile variations in AQ Eri are shorter than even one orbit. The fact that the comparison between the original and the reconstructed data shows evidence of systematic differences (especially the grey scale plot for set $1 \mathrm{~b}$ and the $V / R$ plot for set $1 \mathrm{c})$, seems to indicate the latter. 


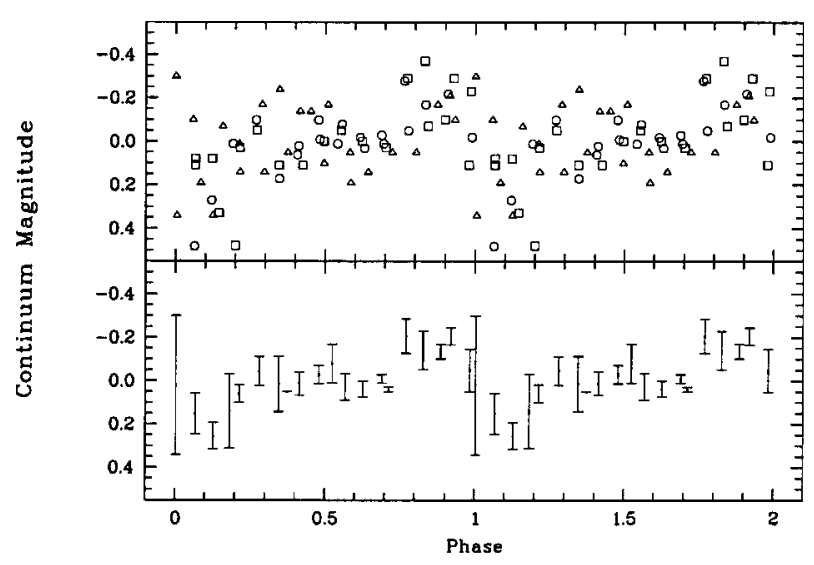

Fig. 3. The $\mathrm{H} \alpha$ continuum magnitude of the VW Hyi data as a function of phase. The upper plot has been composed out of data sets 1 (o), $2(\triangle)$, and $3(\square)$, with the respective average values subtracted. The lower plot gives those three data sets averaged into bins of 0.05 orbital phases, with the error bars referring to $\pm 1 \sigma$ deviation of the averaging process. The data are repeated to show two orbits.

\subsubsection{VW Hyi}

The maps show an evolution of the emission distribution from a combined secondary star/gas stream location in set 1 to a dominating bright spot in set 3 (Figs. A.12-A.14). The ring-shaped disc signature is present, but - like in WX Cet - distorted due to the low $\mathrm{S} / \mathrm{N}$.

While the $V / R$ plot comparison of the original and the reconstructed data are in good agreement with each other, there are some systematic differences to be seen in the trailed spectrograms especially for set 1 . This concerns a narrow emission component that is stronger in the reconstructed data from phases 0.7 to 1.2 and weaker during the other phases. We attribute this to an occultation effect of this emission component, the latter coming most probably from the gas stream or the secondary star (which shows us its non-irradiated backside around phase 0.0). This violates one of the prerequisites for Doppler mapping, specifically that all emission sources have to be visible at all phases. We find a similar phenomenon for TU Men (Sect. 4.3.6).

While we did not detect any systematic variation in the $\mathrm{H} \alpha$ equivalent widths, the continuum - i.e. the optically thick emission - shows a hump at phases $0.75-1.0$ - probably from the gas stream -, followed by a shallow eclipse up to phase 1.2 (Fig. 3$)^{2}$. Although the behaviour of the continuum may be different from that of the emission line, this indicates that the inclination of the system is high enough for occultation effects. Also the fact that the differences in the trailed spectrograms decrease from set 1 to set 3 , while at the same time the Doppler maps show that emission from the secondary star becomes less dominant in this sequence, supports the interpretation that the narrow emission component originates from the secondary star and is occulted during the corresponding phases.

\footnotetext{
2 The uncertainties in the determination of the continuum (which was measured with a graphics cursor) are hard to quantify, but the fact that all three data sets show the same basic features at identical phases proves the reality of this variation.
}

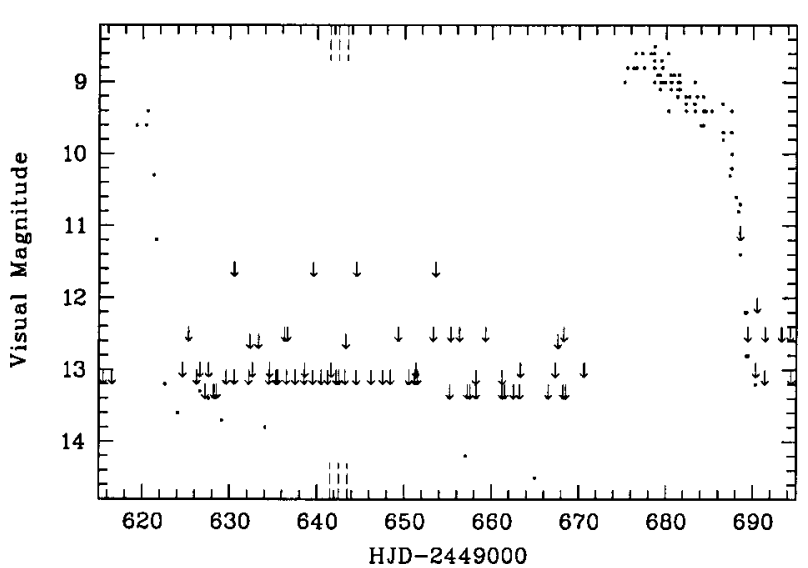

Fig. 4. Long-term light curve for VW Hyi based on AAVSO observations (Mattei 1999, private communication). Upper brightness limits are marked by arrows. The dates of the spectroscopic measurements are indicated by the dashed lines.

The data presented here have been taken 21 days after maximum of a normal outburst, with the system apparently being in a long, slow decline (Fig. 4). The phenomena reported here might therefore either represent a continuous evolution from the outburst to the quiescent state, or a short-term fluctuation (timescale of a few days).

\subsubsection{RZ Leo}

Both data sets yield very similar emission distributions in the form of two maxima on the leading and on the trailing side of the disc (Figs. A.16 and A.17), with the latter maximum being the stronger one. The intensity difference appears to be more pronounced in data set 2, but it remains unclear if this variation is significant or due to noise.

In view of the spotty appearance of other Doppler maps in this atlas (WX Cet, VW Hyi) it is justified to ask if the spots in RZ Leo might be artefacts as well. However, in this case, also the trailed spectrograms show the unambiguous presence of an additional component. At least the spectrogram for data set 1 furthermore shows evidence in the form of a double wave for a second component. While the second spot (on the leading side) in data set 2 might therefore be questionable, in set 1 the strength of this feature appears to exclude an artificial origin.

\subsubsection{TU Men}

The Doppler map of TU Men shows a strong emission feature from the secondary star and otherwise pure disc emission (Fig. A.19). The differences between the reconstructed and the original data which can be seen in the grey scale plots affect the same phases as in the case of VW Hyi (Sect. 4.3.4). Also the explanation is the same: if an emission feature is not visible at all phases (i.e. in violation of one of the prerequisites for Doppler tomography), the Doppler fit will smear the signal over all phases. The feature will therefore be artificially enhanced during the phases when it is absent in the original data 
(here: phases $0.8-1.2$ ), and it will be reconstructed with diminished strength in the phases where it is visible (here: $0.3-0.7)^{3}$.

However, while for TU Men the residuals are more positive than negative, the VW Hyi data set 1 shows the opposite behaviour. In other words, in TU Men the IES feature is diminished rather than enhanced, while in VW Hyi the enhancement is stronger.

A possible reason could be a much stronger obscuration in VW Hyi. Indeed the differences in the strength of the emission feature at the respective phases appear to be more pronounced in VW Hyi than in TU Men. Unfortunately, the TU Men data had not been flux-calibrated so that the strength of a possible obscuration effect in TU Men cannot be compared to that in VW Hyi.

\subsubsection{HS Vir}

The Doppler fitting resulted in a dominant emission feature in the upper left quadrant of the map (Fig. A.21). However, both in strength and in shape there are significant residuals when comparing the reconstructed with the original data. Possible reasons are either an obscuration effect like in VW Hyi (Sect. 4.3.4) or sub-orbital variations like probably in AQ Eri (Sect. 4.3.3). A comparison of the line profiles of the individual phase bins (Fig. A.20) makes the second possibility appear more probable. A good example is phase 0.15 , where the line profile over two orbits (left and right plot) has changed both in its general shape and even in the width of its wings. Furthermore, Howell et al. (1990) found strong variations on non-orbital time scales in the light curve of HS Vir, that could not be explained by flickering. In this case, Doppler mapping with respect to the orbital period yields no representative image of the emission distribution. It might be possible that this technique can be applied successfully, if a very large data set is used, so that non-orbital variations are averaged out.

\section{Discussion}

The systems analysed in this paper have in common that they are all of the SU UMa subclass, i.e. they are dwarf novae with orbital periods (in this case) ranging between 1.4 and $2.8 \mathrm{~h}$, and they have low mass-transfer rates resulting in comparatively faint, optically thin, accretion discs. Excluding the extremely long periodic system TU Men, the period range spanned by the systems even amounts to only $27 \mathrm{~min}$. It is therefore rather surprising that in these 7 systems with supposedly common basic properties we are confronted with almost the complete variety of isolated emission sources (IES).

One can now attempt to look for specific differences between the systems regarding their parameters. In general, there appears to be a slight dependence for the IES on the inclination $i$ of the $\mathrm{CV}$, in that emission from the trailing side of the $\operatorname{disc}\left(v_{x}<0\right.$; bright spot, etc.) is more likely to be present in high-inclination systems, and emission from the leading side is

\footnotetext{
${ }^{3}$ An extension of the Doppler tomography method to account for emission components with variable strength has been very recently proposed by Steeghs (2003).
}

more frequent in low-inclination objects (Tappert \& Hanuschik 2001). Other possibly interesting parameters are the mass ratio $q=M_{2} / M_{1}$, which contains information on the stellar components, and the outburst recurrence time $t_{\text {rec }}$, which is related to the transfer and accretion process. Most of these parameters directly or indirectly affect the equivalent width $W_{\mathrm{H} \alpha}$ of the line. If the same mixture of effects is also responsible for the emission distribution, one should see a corresponding correlation. Finally, the orbital period $P_{\text {orb }}$ is indicative of the mass of the secondary star.

We have listed these parameters together with the detected IES types in Table 3 . With the range in $P_{\text {orb }}$ being rather narrow, we do not expect it to play an important role in the IES distribution for the current sample. Instead the equivalent width seems to qualify as a better sorting criterion. It is influenced by a mixture of system (e.g. inclination) and disc (e.g. accretion rate) dependent parameters, which is likely to be also the case for the emission distribution. As all data sets for a specific system show in general similar additional emission components, we have sorted the data in Table 3 according to the average equivalent width of a system.

Still, there does not appear to exist any clear correlation with the IES type. For example, emission from the trailing side $\left(v_{x}<0\right)$ is observed almost over the whole range of $W_{\mathrm{H} \alpha}$, while emission from the secondary star (TU Men) is right in the middle of our sample. However, we note that emission from the leading side is only present in two systems with $W_{\mathrm{H} \alpha}>100 \AA$ (RZ Leo \& WX Cet). A look at the only other parameter that is roughly known for all the systems, the recurrence time, seemingly strengthens this tendency, since these two WZ Sge related systems represent the only ones that exclusively show superoutbursts. However this very probably is an artifical effect due to the smallness of our sample. IES on the leading side have also been at least suspected ${ }^{4}$ e.g. in SU UMa (Thorstensen et al. 1986) and CY UMa (Martínez-Pais \& Casares 1995), which both show also normal outbursts and have recurrence times much less than one year (Ritter \& Kolb 1998).

For the other parameters the list is largely incomplete. This is basically due to the fact that the calculation of $i$ and $q$ needs some knowledge about the secondary star, the latter being difficult to detect in short-period CVs. Furthermore, those parameters are usually rather uncertain with $1 \sigma$ errors in the order of 10 to $20 \%$.

An additional problem is that we are always dealing with a combination of parameters, and a sample of 7 systems is certainly too small to provide significant statistics in order to separate the individual influences. So, while the variety of the phenomena detected here might come as a surprise, the lack of a clear correlation with the system parameters probably should not.

Perhaps the only system whose emission distribution can be fixed to a parameter is TU Men, which shows a strong contribution from the secondary star in its quiescent state. In SU UMa

\footnotetext{
${ }^{4}$ Doppler tomography has been published for none of these systems, so that the evidence on their emission distribution is only secondary. This emphasizes the need for more detailed line-profile studies for short period CVs.
} 
Table 3. Isolated emission features detected in the individual data sets, which here appear sorted with increasing equivalent width. Columns 2 to 4 give basic parameters of the systems, i.e. the period, the inclination, the mass ratio $q=M_{2} / M_{1}$, and the (normal) outburst recurrence time, except for RZ Leo and WX Cet, which show only superoutbursts. These parameters were taken from the Ritter \& Kolb (1998) catalogue except where indicated. Columns 5 and 6 contain the data set specific information on the equivalent width of the H $\alpha$ emission line and the accretion state during the observations $(\mathrm{d}=$ decline, $\mathrm{q}=$ quiescence), respectively. The $x$-velocities in Cols. 7 and 8 refer to the emission maxima in the respective halves of the Doppler maps, other emission regions are denoted by their identification. The presence of a feature is indicated by " + ", its absence by “-”, uncertain ones by "??", and very uncertain ones by "??".

\begin{tabular}{lcrrrrrrrrrr}
\hline \hline Data set & $\begin{array}{l}P_{\text {orb }} \\
{[\mathrm{h}]}\end{array}$ & $\begin{array}{r}i \\
{[\mathrm{deg}]}\end{array}$ & $q$ & $\begin{array}{r}t_{\text {rec }} \\
{[\mathrm{d}]}\end{array}$ & $\begin{array}{r}W_{\mathrm{H} \alpha} \\
{[\AA]}\end{array}$ & State & $-v_{x}$ & $+v_{x}$ & $\begin{array}{r}\text { Secondary } \\
\text { star }\end{array}$ & $\begin{array}{c}\text { Gas } \\
\text { stream }\end{array}$ & Fig. \\
\hline AK Cnc & 1.62 & $36^{1}$ & $0.28^{1}$ & $47^{5}$ & -28 & $\mathrm{~d}$ & - & - & - & - & A.2 \\
HS Vir & 1.85 & - & $0.22^{6}$ & 8 & -36 & $\mathrm{q}$ & + & - & - & - & A.21 \\
VW Hyi 1 & 1.78 & $\sim 48^{7}$ & $\sim 0.14^{7}$ & 27 & -42 & $\mathrm{q}$ & - & - & $?$ & + & A.12 \\
VW Hyi 2 & & & & & -40 & $\mathrm{q}$ & + & - & $? ?$ & + & A.13 \\
VW Hyi 3 & & & & & -51 & $\mathrm{q}$ & + & - & - & + & A.14 \\
TU Men & 2.81 & $52^{4}$ & $0.46^{4}$ & 37 & -118 & $\mathrm{q}$ & - & $? ?$ & + & - & A.19 \\
RZ Leo 1 & 1.84 & - & - & $>365^{2}$ & -139 & $\mathrm{q}$ & + & + & - & $? ?$ & A.16 \\
RZ Leo 2 & & & & & -118 & $\mathrm{q}$ & + & $?$ & - & $? ?$ & A.17 \\
AQ Eri 1a & 1.46 & - & - & 78 & -139 & $\mathrm{q}$ & $?$ & - & $? ?$ & $? ?$ & A.8 \\
AQ Eri 1b & & & & & -129 & $\mathrm{q}$ & + & - & $? ?$ & $? ?$ & A.9 \\
AQ Eri 1c & & & & & -131 & $\mathrm{q}$ & + & - & $? ?$ & $? ?$ & A.10 \\
WX Cet 1 & 1.40 & $\leq 65^{3}$ & $\geq 0.11^{3}$ & 450 & -184 & $\mathrm{q}$ & $?$ & $?$ & $? ?$ & - & A.4 \\
WX Cet 2 & & & & & -198 & $\mathrm{q}$ & $? ?$ & + & $? ?$ & - & A.5 \\
WX Cet 3 & & & & & -183 & $\mathrm{q}$ & - & + & $? ?$ & - & A.6 \\
\hline
\end{tabular}

References: ${ }^{1}$ Arenas \& Mennickent (1998), ${ }^{2}$ Ishioka et al. (2001), ${ }^{3}$ Mennickent (1994), ${ }^{4}$ Mennickent (1995a), ${ }^{5}$ Mennickent et al. (1996), ${ }^{6}$ Mennickent et al. (1999), ${ }^{7}$ Tappert (1999).

systems this phenomenon has been hitherto observed only in outburst. It appears that in this respect TU Men already qualifies as a long-periodic system, whose disc is bright enough to induce emission on the surface of the secondary star.

Finally, we would like to point out that at least in one system the variations between different data sets are not irregular. The analysis of spectra from subsequent nights of VW Hyi show a clear long-term evolution from almost pure gas-stream emission to a dominant bright spot. At the time of the observations, the system apparently is in a state of a prolonged, slow decline (with a rate of roughly 1 mag in 40 days) about 20 days after a normal outburst and $\sim 35$ days before a superoutburst (Fig. 4). The long-term variations in the line profile therefore take place during a period where the overall brightness of the system is barely changing. Apparently even variations on small scales - in the sense that they are not accompanied or immediately followed by large photometric variations (i.e., outbursts) - of the mass-transfer rate and/or in the disc itself yield significant changes in the line profile.

\section{Conclusions}

The here presented atlas of line-profile analyses for 7 SU UMatype dwarf novae increases the number of available Doppler tomographies for such systems by almost $100 \%$. Our maps certainly suffer from the low quality of the data with respect to spectral resolution and low $\mathrm{S} / \mathrm{N}$. The reason for the former is that the data were not taken with the aim to perform this kind of analysis, but rather to derive the most basic parameter for $\mathrm{CVs}$, the orbital period. Nevertheless, they still served to gain a basic idea on the occurrence of isolated emission sources (IES) in these systems.

The noisy appearance of the maps, on the other hand, is mostly due to the fact that several systems showed non-orbital variations of the emission distribution, with the consequence that the respective data sets could not be combined in order to improve the $\mathrm{S} / \mathrm{N}$, thus actually representing one of the results of our study. Apart from that we can draw the following conclusions:

1. There is a large variety of IES phenomena present even in systems with in principle very similar physical properties.

2. With HS Vir and probably also AQ Eri we find two systems which show sub-orbital variations in their line profile. Consequently, Doppler tomography does not yield a representative image of the emission distribution in these cases. Very large data sets, where this type of variation can be averaged out, might be needed for this purpose. These systems also represent interesting targets for the recently introduced approach to map the emission line flickering (Diaz 2001).

3. On the other hand, the non-orbital variations in VW Hyi occur on long-term timescales and therefore represent a trend in the evolution of the emission distribution. Here, long-term monitoring of the inter-outburst state via Doppler mapping has the potential to provide important clues on the outburst mechanisms (e.g. the role of irradiation).

4. TU Men can now claim the record of being the CV with the shortest orbital period (and the only SU UMa dwarf nova) to show emission from the secondary star in quiescence ${ }^{5}$. Previously, this was the nova-like DW UMa $\left(P_{\text {orb }}=3.28 \mathrm{~h}\right)$, which showed this type of emission in its low state (Dhillon et al. 1994).

${ }^{5}$ Emission from the secondary star has possibly also been detected in the SU UMa dwarf nova OU Vir $\left(P_{\text {orb }}=1.75 \mathrm{~h}\right)$ by Mason et al. (2002). However, the authors are very cautious with their interpretation due to the problematic definition of the fiducial phase in this system. 
5. The re-examination of "old" data with new techniques is important and meriting, even if only to motivate more detailed studies on the respective systems.

Acknowledgements. We thank the anonymous referee for careful reading and thoughtful comments, which helped to improve this paper. We also thank Marcos Diaz for enlightening discussions on lineprofile variations. The VW Hyi data were taken by Walter Wargau $(† 1996)$. RM acknowledges support by FONDECYT 1000324.

\section{Appendix A: The atlas}
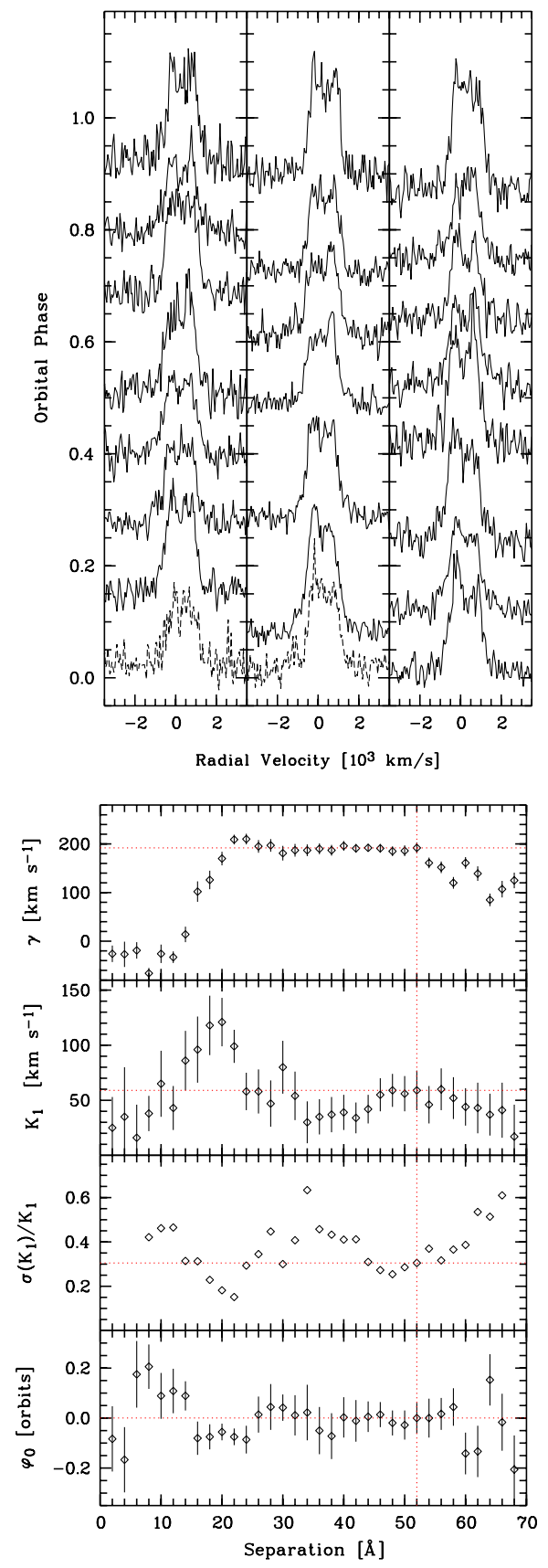

Fig. A.3. Top: line profiles of selected $10 \%$ phase bins for WX Cet. Data sets 1-3 are plotted from left to right. The time sequence within one data set is symbolised by the sequence solid - dashed. Bottom: diagnostic diagram for the combined data set of WX Cet, already corrected for the derived zero phase. The dotted lines mark the chosen separation and the corresponding parameters.
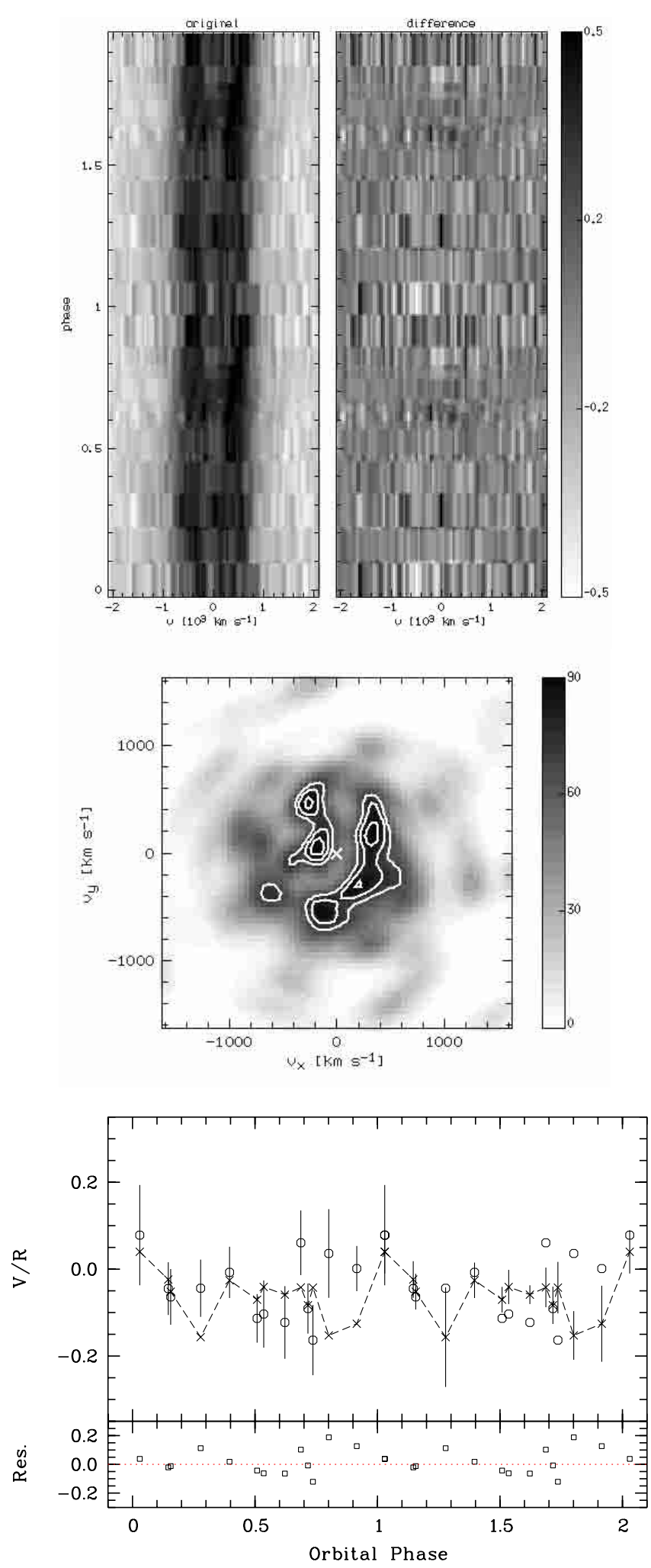

Fig. A.4. Doppler Tomography for WX Cet, data set 1. Top: original (left) and difference (original-reconstructed; right) spectrum. The intensity bar on the right refers to the latter plot. Middle: doppler map. Contour levels are at 86,77 , and 68 per cent of the maximum intensity. Bottom: $V / R$ plot of the original $(\circ)$ and the reconstructed $(\times$ and dashed line) data, and the residuals (bottom of the plot). In phases 0 to 1 , error bars are given for the original data, phases 1 to 2 show those for the reconstructed data. 

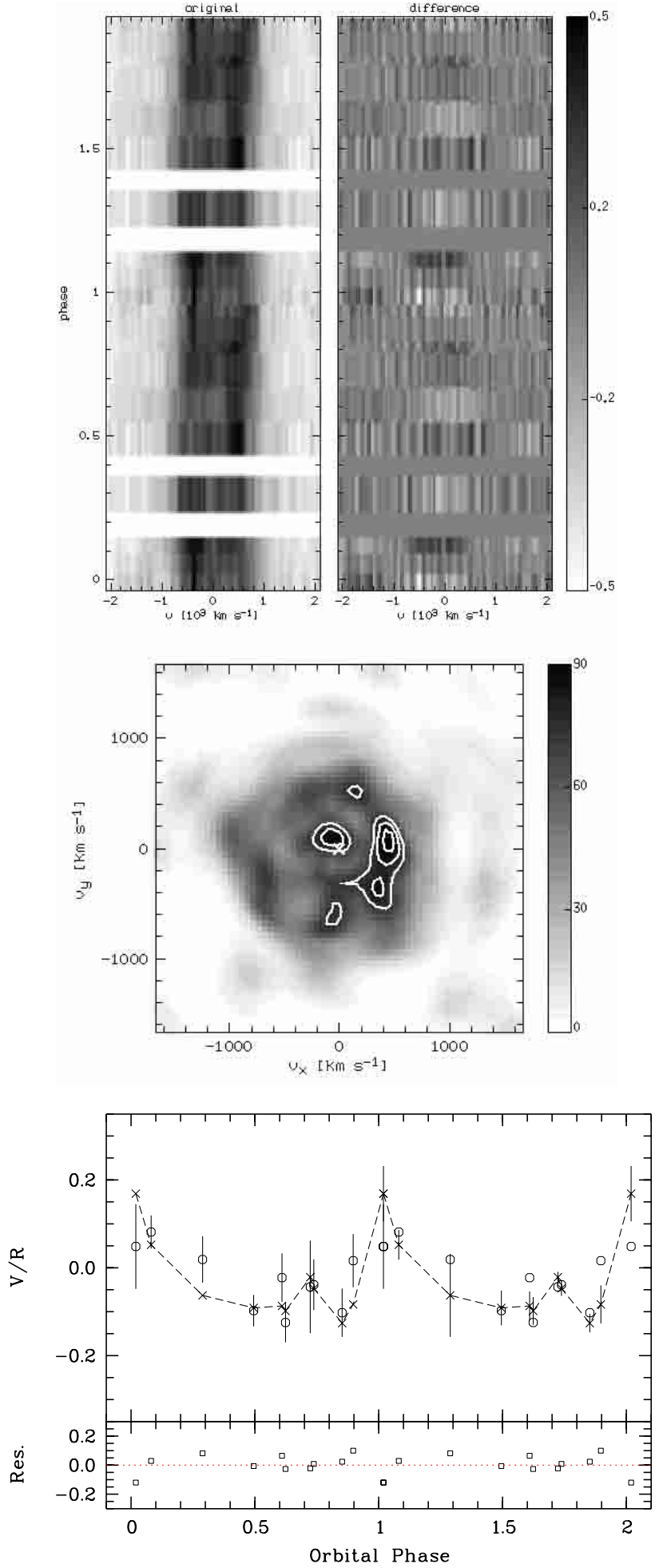

Fig. A.5. Doppler Tomography for WX Cet, data set 2. Top: original (left) and difference (original-reconstructed; right) spectrum. The intensity bar on the right refers to the latter plot. Middle: doppler map. Contour levels are at 90, 81, and 72 per cent of the maximum intensity. Bottom: $V / R$ plot of the original ( $\circ$ ) and the reconstructed $(\times$ and dashed line) data, and the residuals (bottom of the plot). In phases 0 to 1 , error bars are given for the original data, phases 1 to 2 show those for the reconstructed data.
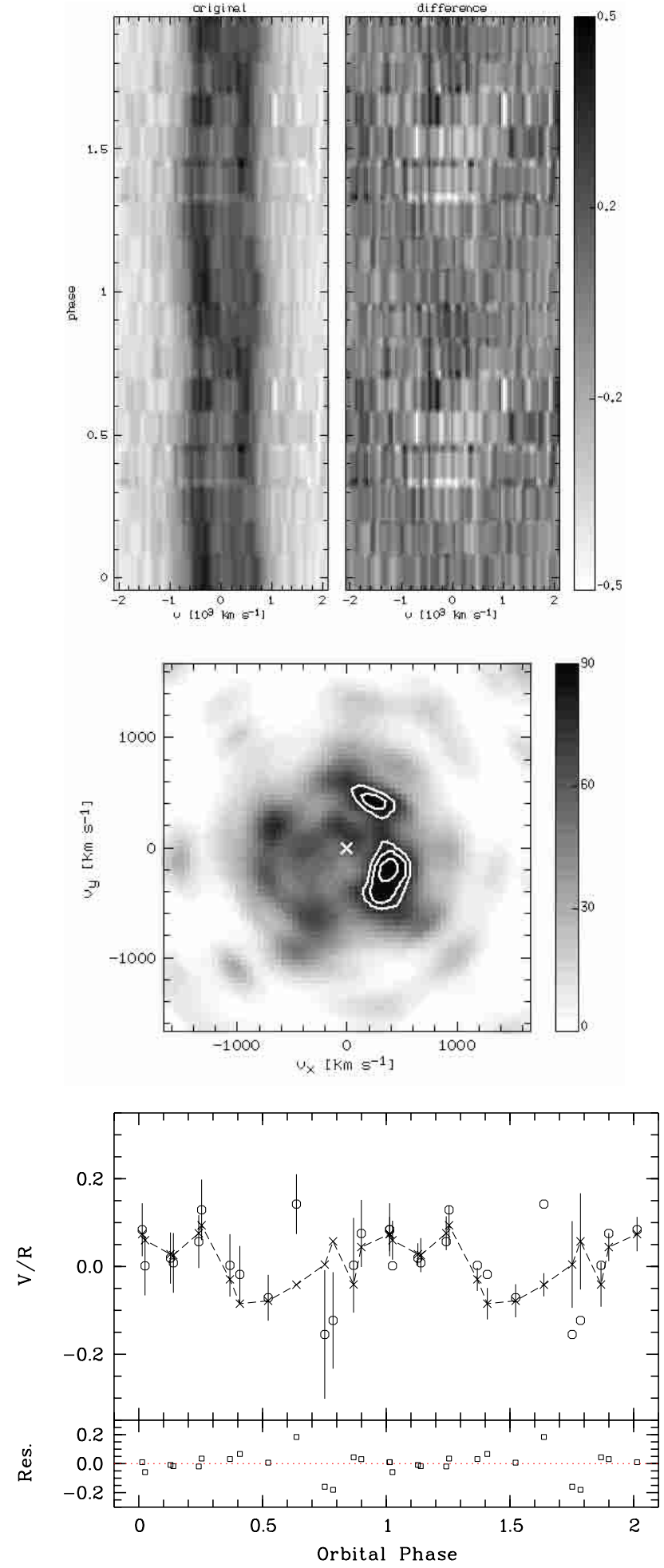

Fig. A.6. Doppler Tomography for WX Cet, data set 3. Top: original (left) and difference (original-reconstructed; right) spectrum. The intensity bar on the right refers to the latter plot. Middle: doppler map. Contour levels are at 93, 84, and 75 per cent of the maximum intensity. Bottom: $V / R$ plot of the original ( $\mathrm{o}$ ) and the reconstructed $(\times$ and dashed line) data, and the residuals (bottom of the plot). In phases 0 to 1 , error bars are given for the original data, phases 1 to 2 show those for the reconstructed data. 


\section{References}

Arenas, J., \& Mennickent, R. E. 1998, A\&A, 337, 472

Dhillon, V. S., Jones, D. H. P., \& Marsh, T. R. 1994, MNRAS, 266, 859

Diaz, M. P. 2001, ApJ, 553, L177

Hoard, D. W., Thorstensen, J. R., \& Szkody, P. 2000, ApJ, 537, 936

Horne, K. 1995, A\&A, 297, 273

Horne, K., \& Marsh, T. M. 1986, MNRAS, 218, 761

Howell, S. B., Szkody, P., Kreidel, T. J., Mason, K. O. \& Puchnarewicz, E. M. 1990, PASP, 102, 758

Ishioka, R., Kato, T., Uemura, M., et al. 2001, PASJ, 53, 905

Kaitchuck, R. H., Schlegel, E. M., Honeycutt, R. K., et al. 1994, ApJS, 93, 519

Kaitchuck, R. H., Schlegel, E. M., White II, J. C., \& Mansperger, C. S. 1998, ApJ, 499, 444

Marsh, T. R. 2001, in Astrotomography, Indirect Imaging Methods in Observational Astronomy, ed. H. M. J. Boffin, D. Steeghs, \& J. Cuypers, Lect. Notes Phys., 573, 1

Marsh, T. R., \& Horne, K. 1988, MNRAS, 235, 269

Martínez-Pais, I. G., \& Casares, J. 1995, MNRAS, 275, 699

Martínez-Pais, I. G., Giovannelli, F., Rossi, C., \& Gaudenzi, S. 1994, A\&A, 291, 455

Martínez-Pais, I. G., Giovannelli, F., Rossi, C., \& Gaudenzi, S. 1996, A\&A, 308, 833

Mason, E., Howell, S. B., Szkody, P., et al. 2002, A\&A, 396, 633

Mattei, J. A. 1999, Observations from the AAVSO International Database, private communication

Mennickent, R. E. 1994, A\&A, 285, 979

Mennickent, R. E. 1995a, A\&A, 294, 126
Mennickent, R. E., \& Tappert, C. 2001, A\&A, 372, 563

Mennickent, R. E. 1995b, Ph.D. Thesis, Pontificia Universidad Católica de Chile

Mennickent, R. E., Nogami, D., Kato, T., \& Worraker, W. 1996, A\&A, 315,493

Mennickent, R. E., Matsumoto, K. \& Arenas, J. 1999, A\&A, 348, 466

North, R., Marsh, T. R., Moran, C. K. J., et al. 2001, in Astrotomography, Indirect Imaging Methods in Observational Astronomy, ed. H. M. J. Boffin, D. Steeghs, \& J. Cuypers, Lect. Notes Phys., 573, 33

Ritter, H., \& Kolb, U. 1998, A\&AS, 129, 83

Schneider, D. P., \& Young, P. 1980, ApJ, 238, 946

Shafter, A. W. 1983a, ApJ, 267, 222

Shafter, A. W. 1983b, Ph.D. Thesis, University of California, Los Angeles

Skidmore, W., Mason, E., Howell, S. B., et al. 2000, MNRAS, 318, 429

Smak, J. 1981, Acta Astron., 31, 395

Spruit, H.C. 1998 [astro-ph/9806141]

Steeghs, D. 2003, MNRAS, in press [astro-ph/0305365]

Steeghs, D., Harlaftis, E. T., \& Horne, K. 1997, MNRAS, 290, L28

Stover, R. J. 1981, ApJ, 248, 684

Tappert, C. 1999, Ph.D. Thesis, Ruhr-Universität Bochum

Tappert, C. \& Hanuschik, R.W. 2001, in Astrotomography, Indirect Imaging Methods in Observational Astronomy, ed. H. M. J. Boffin, D. Steeghs, \& J. Cuypers, Lect. Notes Phys., 573, 119

Thorstensen, J. R., Wade, R. A., \& Oke, J. B. 1986, ApJ, 309, 721

Warner, B. 1995, Cataclysmic Variable Stars (Cambridge University Press) 


\section{Online Material}


C. Tappert et al.: An atlas of line profile studies for SU UMa stars, Online Material p 2
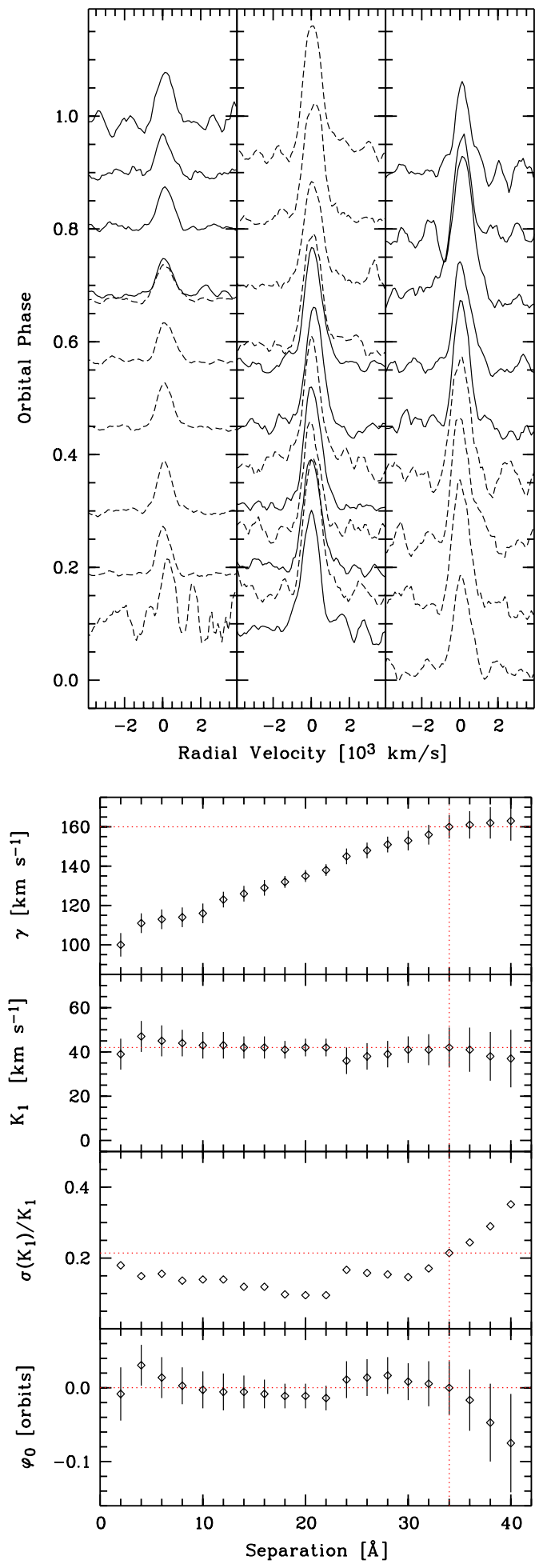

Fig. A.1. Top: line profiles of selected $10 \%$ phase bins for AK Cnc. Data sets 1-3 are plotted from left to right. The time sequence within one data set is symbolised by the sequence solid - dashed. Bottom: diagnostic diagram for AK Cnc, already corrected for the derived zero phase. The dotted lines mark the chosen separation and the corresponding parameters.
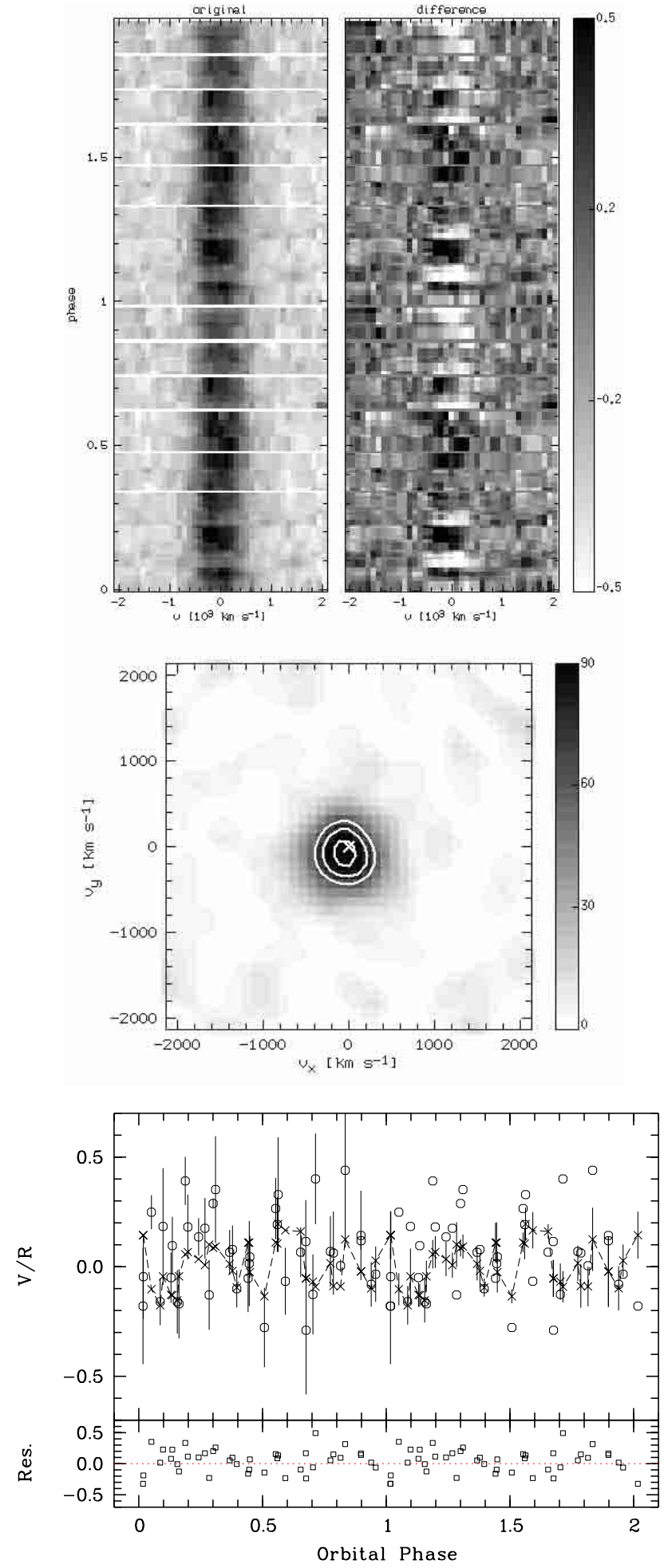

Fig. A.2. Doppler Tomography of AK Cnc. Top: Original (left) and difference (original-reconstructed; right) spectrum. The intensity bar on the right refers to the latter plot. Middle: doppler map. Contour levels are at 90, 75, and 60 per cent of the maximum intensity. Bottom: $V / R$ plot of the original (o) and the reconstructed ( $\times$ and dashed line) data, and the residuals (bottom of the plot). In phases 0 to 1 , error bars are given for the original data, phases 1 to 2 show those for the reconstructed data. 
C. Tappert et al.: An atlas of line profile studies for SU UMa stars, Online Material p 3
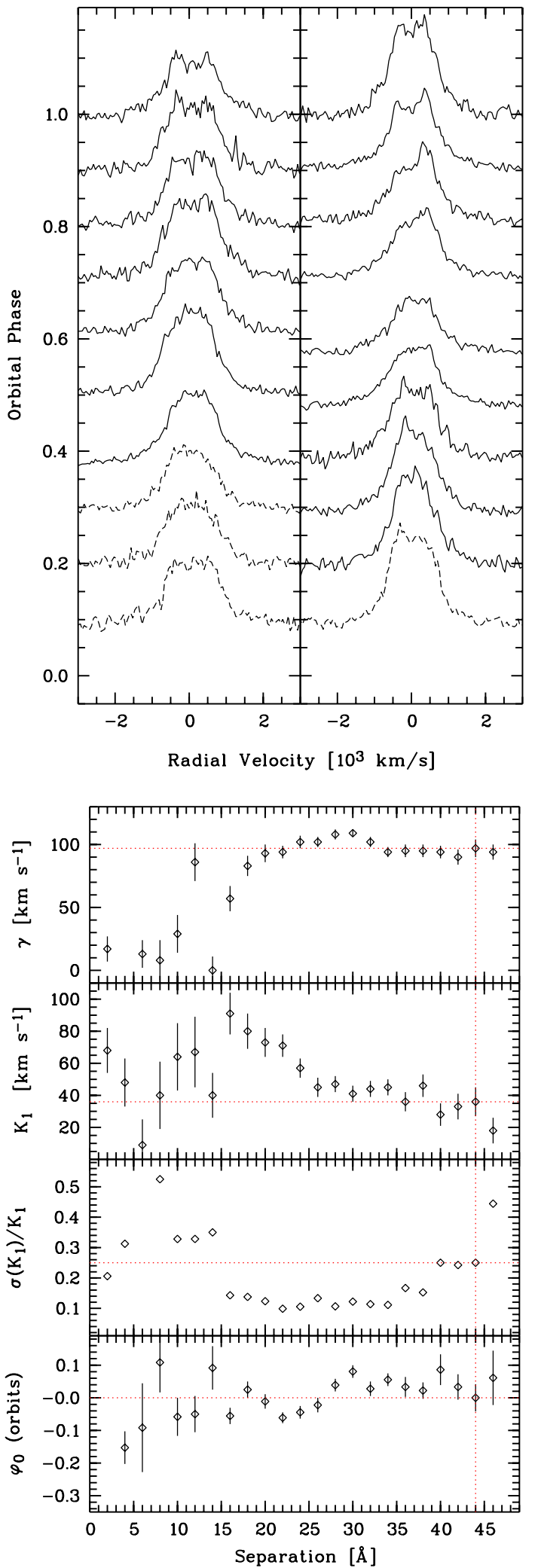

Fig. A.7. Top: line profiles of selected $10 \%$ phase bins for AQ Eri. Data sets $1 \mathrm{a}$ and $1 \mathrm{c}$ are plotted on the left and on the right, respectively. The time sequence within one data set is symbolised by the sequence solid - dashed. Bottom: diagnostic diagram for the combined data set of AQ Eri, already corrected for the derived zero phase. The dotted lines mark the chosen separation and the corresponding parameters.
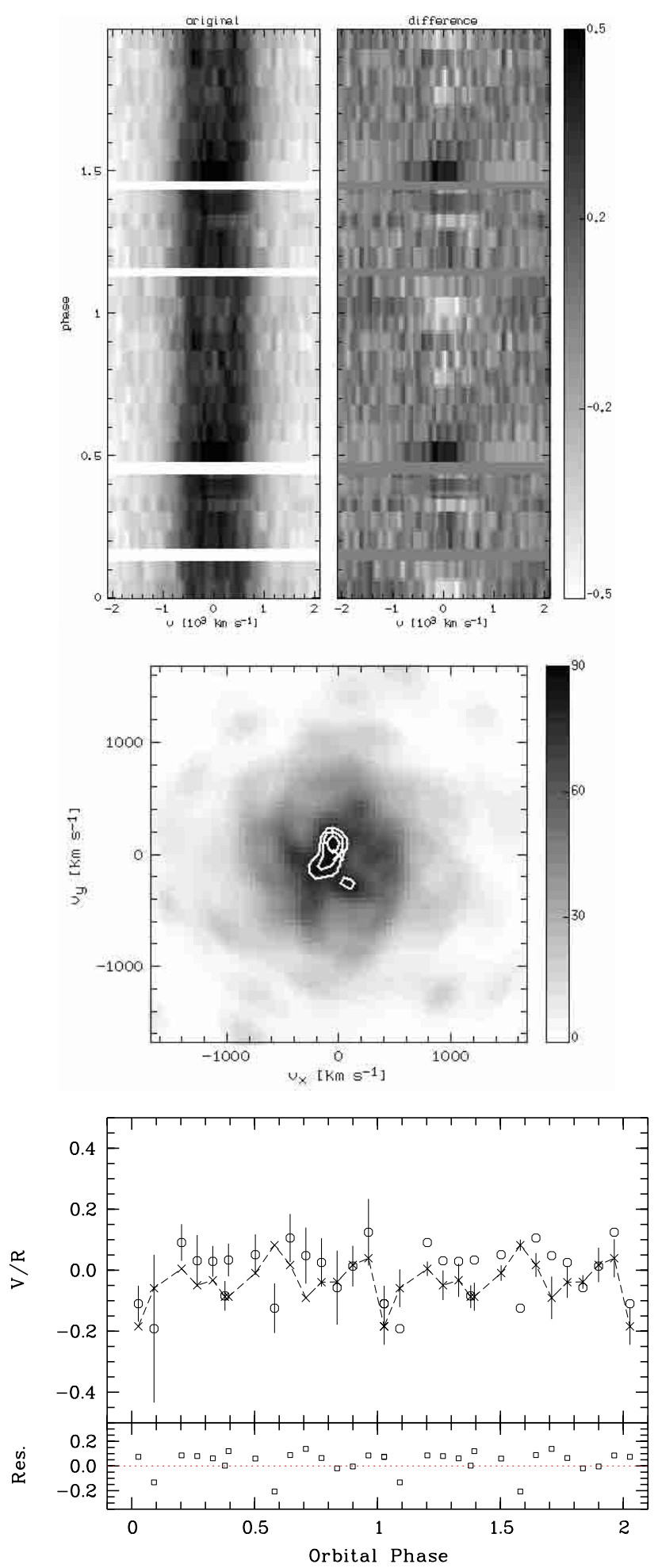

Fig. A.8. Doppler Tomography for AQ Eri, data set 1a. Top: original (left) and difference (original-reconstructed; right) spectrum. The intensity bar on the right refers to the latter plot. Middle: doppler map. Contour levels are at 90, 84, and 78 per cent of the maximum intensity. Bottom: $V / R$ plot of the original $(\circ)$ and the reconstructed $(\times$ and dashed line) data, and the residuals (bottom of the plot). In phases 0 to 1 , error bars are given for the original data, phases 1 to 2 show those for the reconstructed data. 
C. Tappert et al.: An atlas of line profile studies for SU UMa stars, Online Material $p 4$
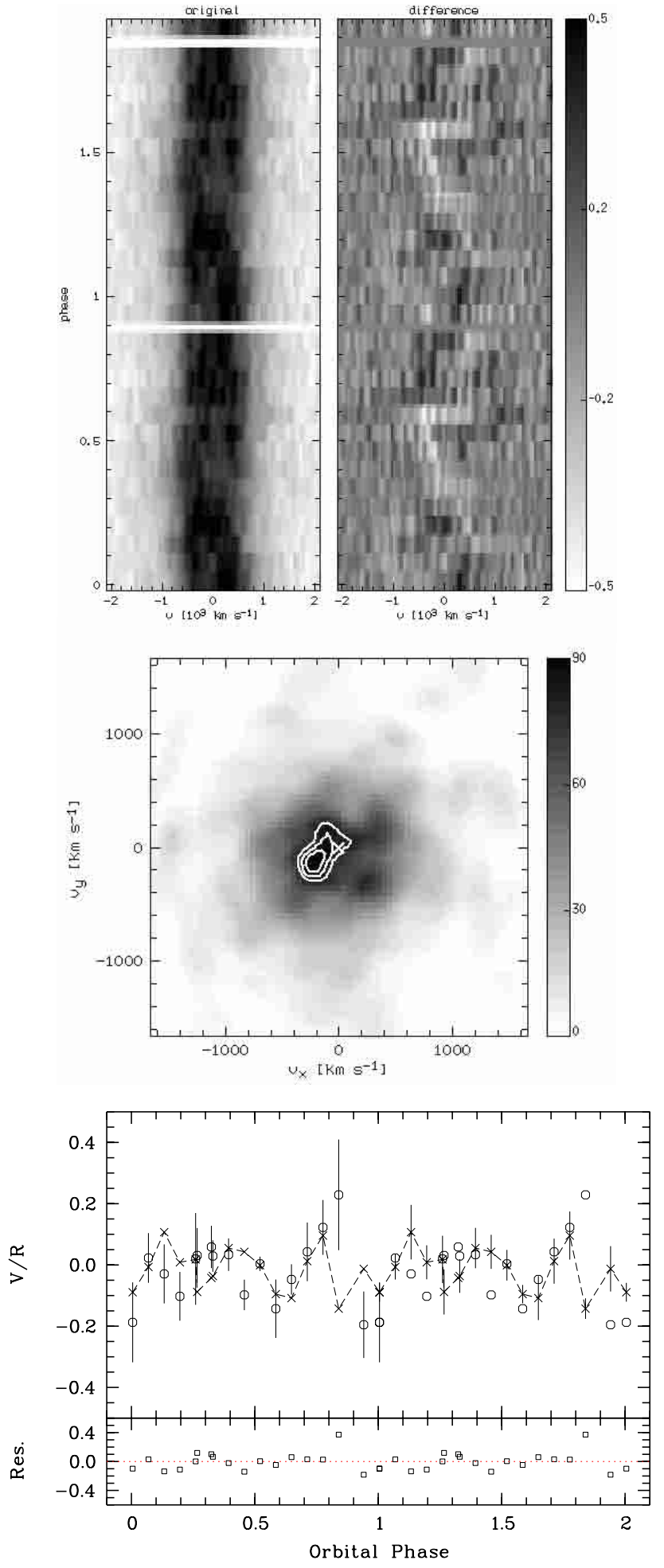

Fig. A.9. Doppler Tomography for AQ Eri, data set 1b. Top: original (left) and difference (original-reconstructed; right) spectrum. The intensity bar on the right refers to the latter plot. Middle: doppler map. Contour levels are at 90, 84, and 78 per cent of the maximum intensity. Bottom: $V / R$ plot of the original ( $\circ$ ) and the reconstructed $(\times$ and dashed line) data, and the residuals (bottom of the plot). In phases 0 to 1 , error bars are given for the original data, phases 1 to 2 show those for the reconstructed data.
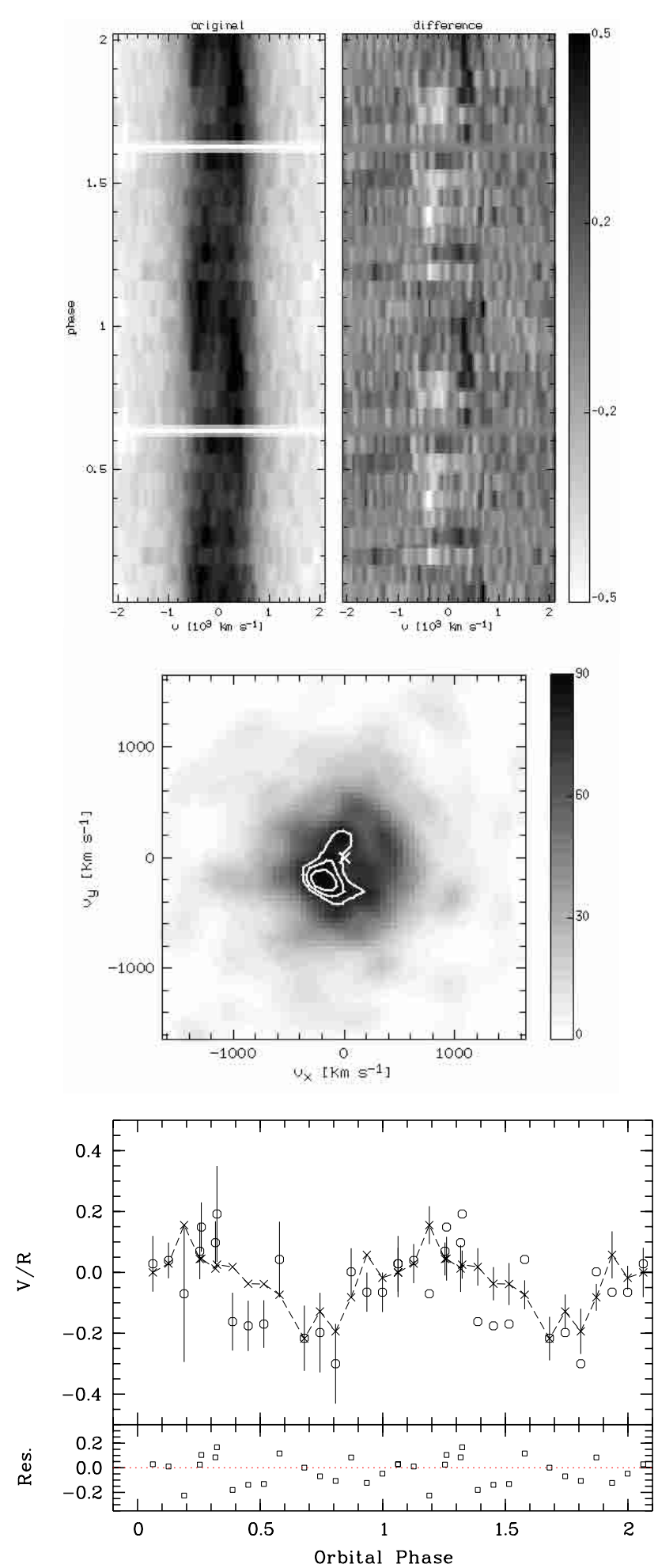

Fig. A.10. Doppler Tomography for AQ Eri, data set 1c. Top: original (left) and difference (original-reconstructed; right) spectrum. The intensity bar on the right refers to the latter plot. Middle: doppler map. Contour levels are at 90, 84, and 78 per cent of the maximum intensity. Bottom: $V / R$ plot of the original ( 0 ) and the reconstructed $(\times$ and dashed line) data, and the residuals (bottom of the plot). In phases 0 to 1 , error bars are given for the original data, phases 1 to 2 show those for the reconstructed data. 
C. Tappert et al.: An atlas of line profile studies for SU UMa stars, Online Material p 5
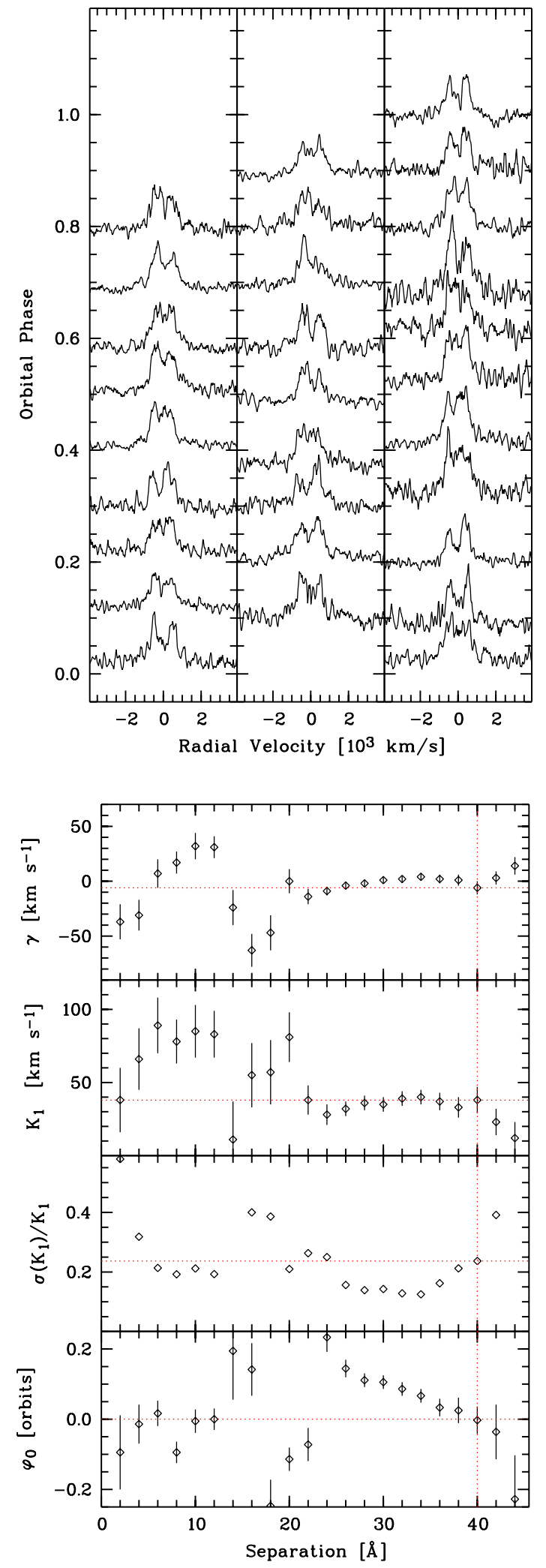

Fig. A.11. Top: line profiles of selected 10\% phase bins for VW Hyi. Data sets $1-3$ are plotted from left to right. Bottom: diagnostic diagram for the combined data set of VW Hyi, already corrected for the derived zero phase. The dotted lines mark the chosen separation and the corresponding parameters.
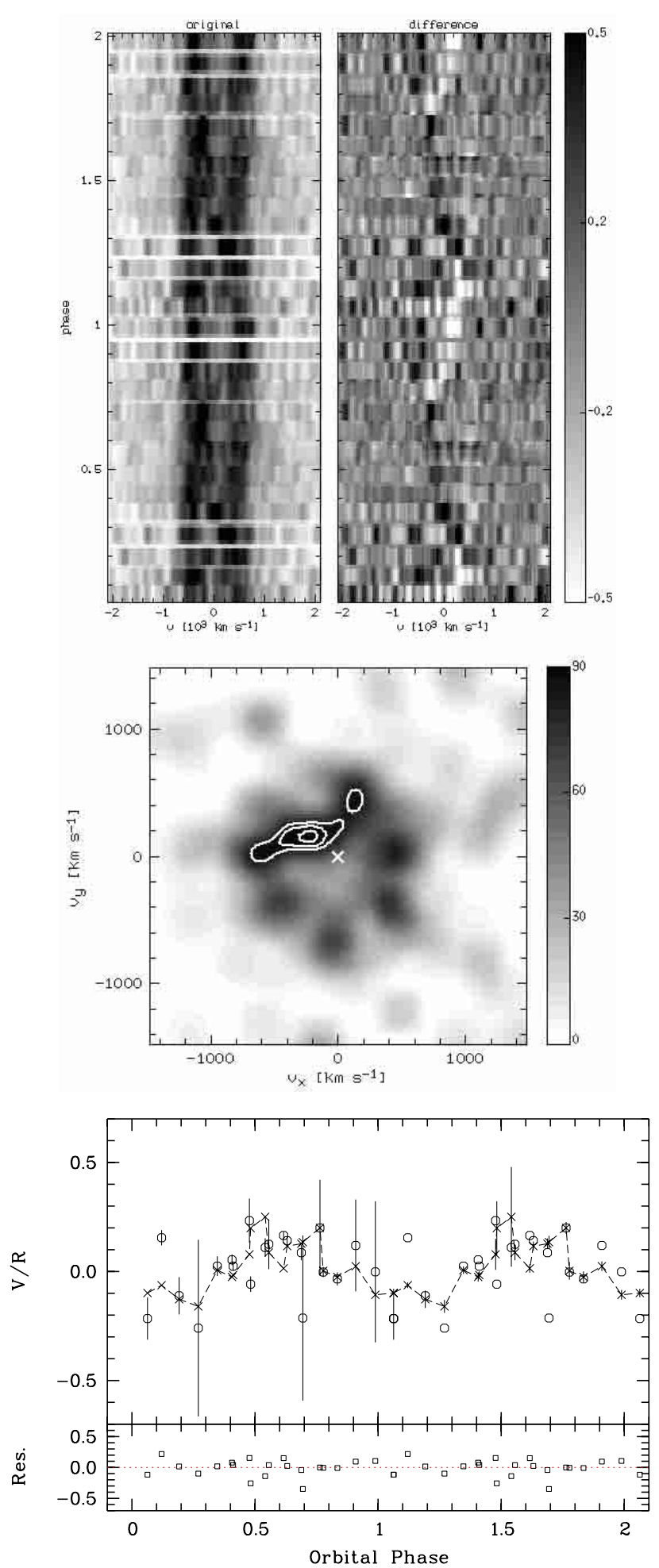

Fig. A.12. Doppler Tomography for VW Hyi, data set 1. Top: original (left) and difference (original-reconstructed; right) spectrum. The intensity bar on the right refers to the latter plot. Middle: doppler map. Contour levels are at 96,89 , and 82 per cent of the maximum intensity. Bottom: $V / R$ plot of the original $(\circ)$ and the reconstructed $(\times$ and dashed line) data, and the residuals (bottom of the plot). In phases 0 to 1 , error bars are given for the original data, phases 1 to 2 show those for the reconstructed data. 
C. Tappert et al.: An atlas of line profile studies for SU UMa stars, Online Material $p 6$
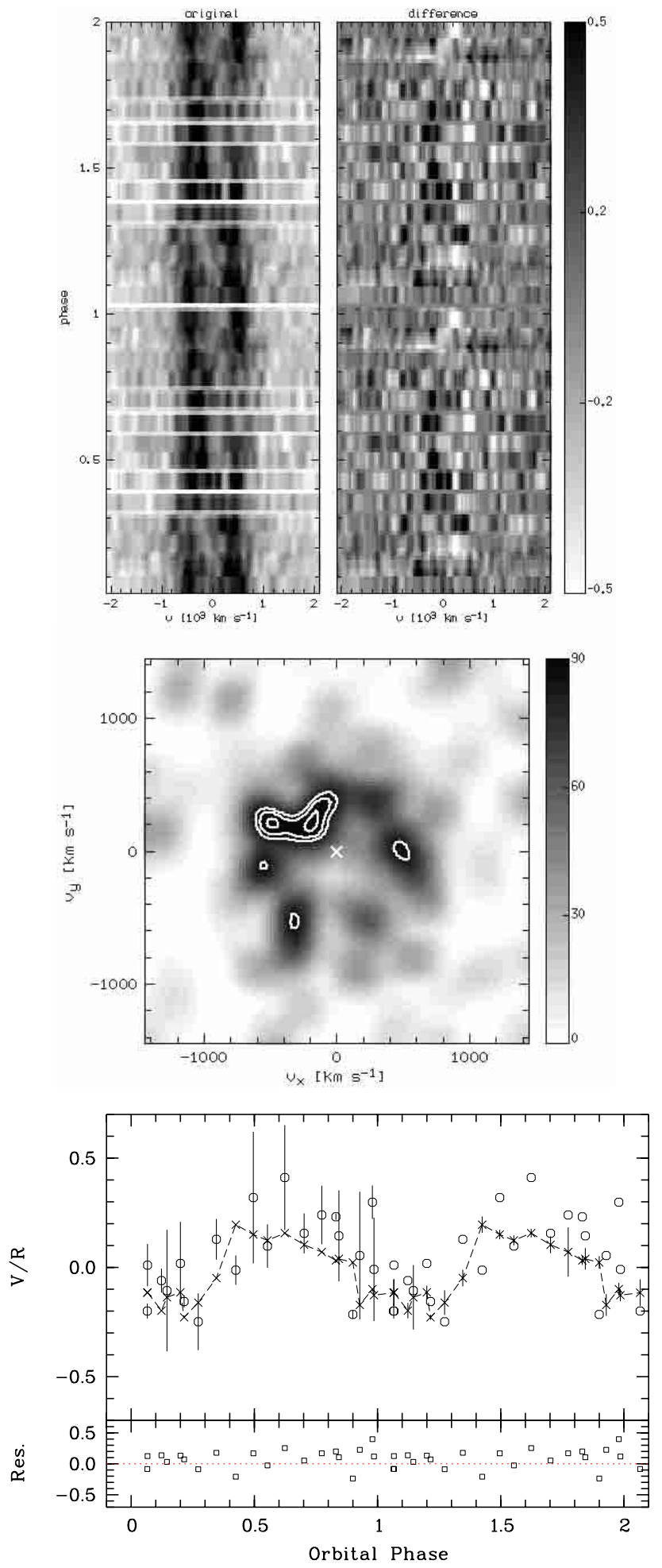

Fig. A.13. Doppler Tomography for VW Hyi, data set 2. Top: original (left) and difference (original-reconstructed; right) spectrum. The intensity bar on the right refers to the latter plot. Middle: doppler map. Contour levels are at 97, 90, and 83 per cent of the maximum intensity. Bottom: $V / R$ plot of the original $(\circ)$ and the reconstructed $(\times$ and dashed line) data, and the residuals (bottom of the plot). In phases 0 to 1 , error bars are given for the original data, phases 1 to 2 show those for the reconstructed data.
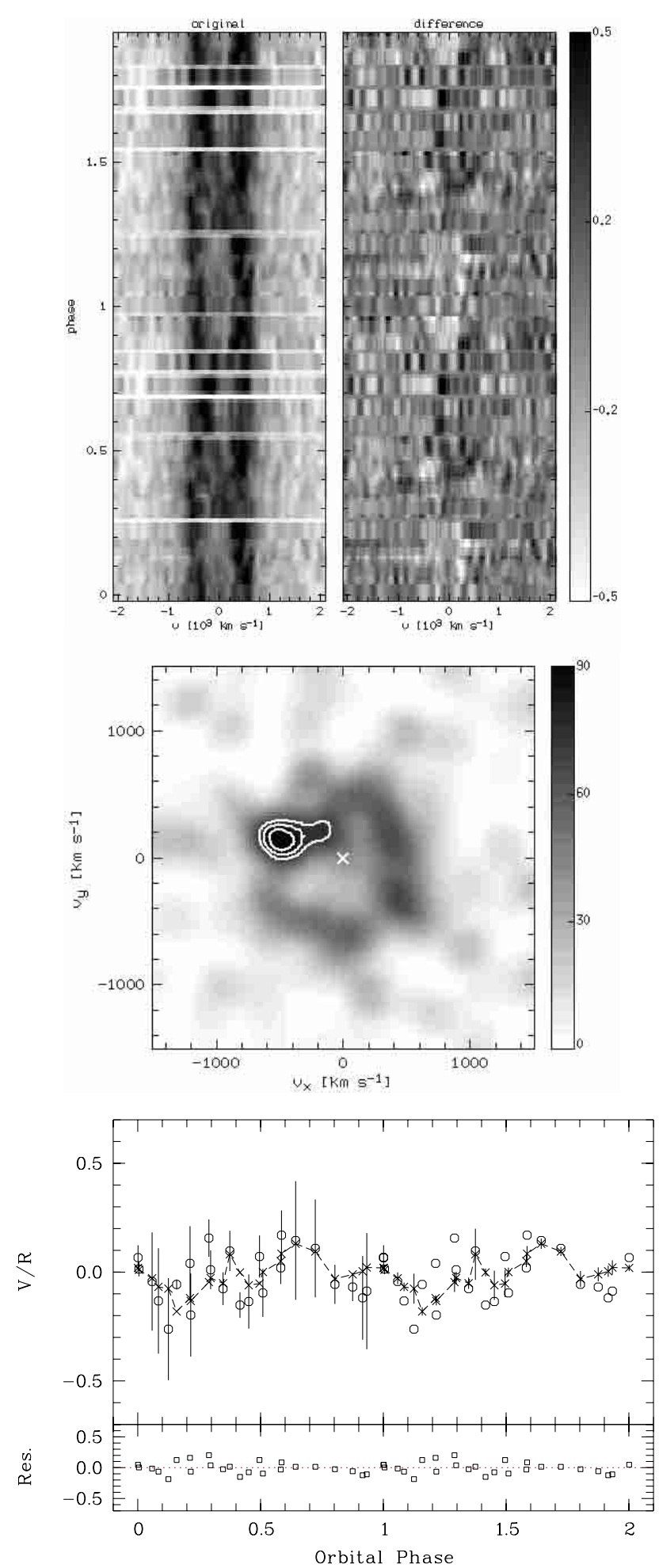

Fig. A.14. Doppler Tomography for VW Hyi, data set 3. Top: original (left) and difference (original-reconstructed; right) spectrum. The intensity bar on the right refers to the latter plot. Middle: doppler map. Contour levels are at 90, 80, and 70 per cent of the maximum intensity. Bottom: $V / R$ plot of the original ( $\mathrm{o}$ ) and the reconstructed $(\times$ and dashed line) data, and the residuals (bottom of the plot). In phases 0 to 1 , error bars are given for the original data, phases 1 to 2 show those for the reconstructed data. 
C. Tappert et al.: An atlas of line profile studies for SU UMa stars, Online Material $p 7$
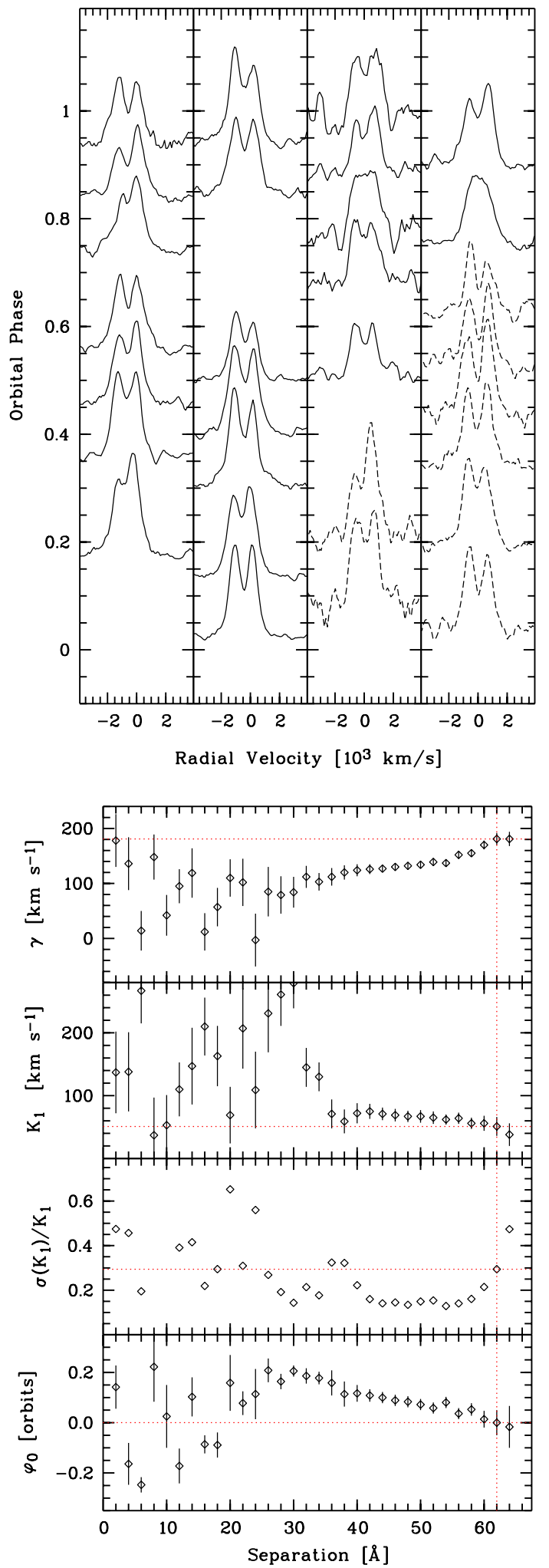

Fig. A.15. Top: line profiles of selected $10 \%$ phase bins for RZ Leo. Data sets $1 \mathrm{a}, 1 \mathrm{~b}, 2 \mathrm{a}, 2 \mathrm{~b}$ are plotted from left to right. The time sequence is symbolised by the line styles solid - dashed. Bottom: diagnostic diagram of RZ Leo, data set 2 , already corrected for the derived zero phase. The dotted lines mark the chosen separation and the corresponding parameters.
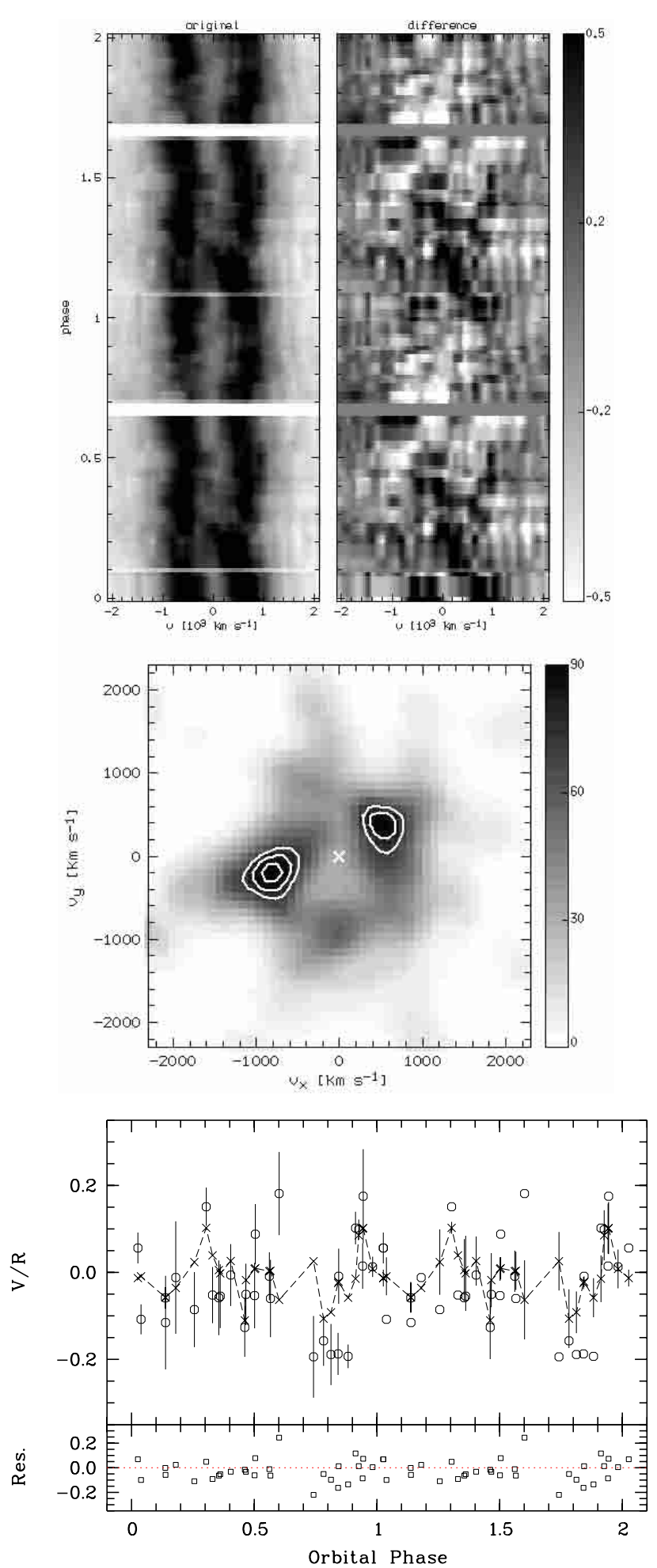

Fig. A.16. Doppler Tomography for RZ Leo, data set 1. Top: original (left) and difference (original-reconstructed; right) spectrum. The intensity bar on the right refers to the latter plot. Middle: doppler map. Contour levels are at 90, 80, and 70 per cent of the maximum intensity. Bottom: $V / R$ plot of the original $(\circ)$ and the reconstructed $(x$ and dashed line) data, and the residuals (bottom of the plot). In phases 0 to 1 , error bars are given for the original data, phases 1 to 2 show those for the reconstructed data. 
C. Tappert et al.: An atlas of line profile studies for SU UMa stars, Online Material p 8
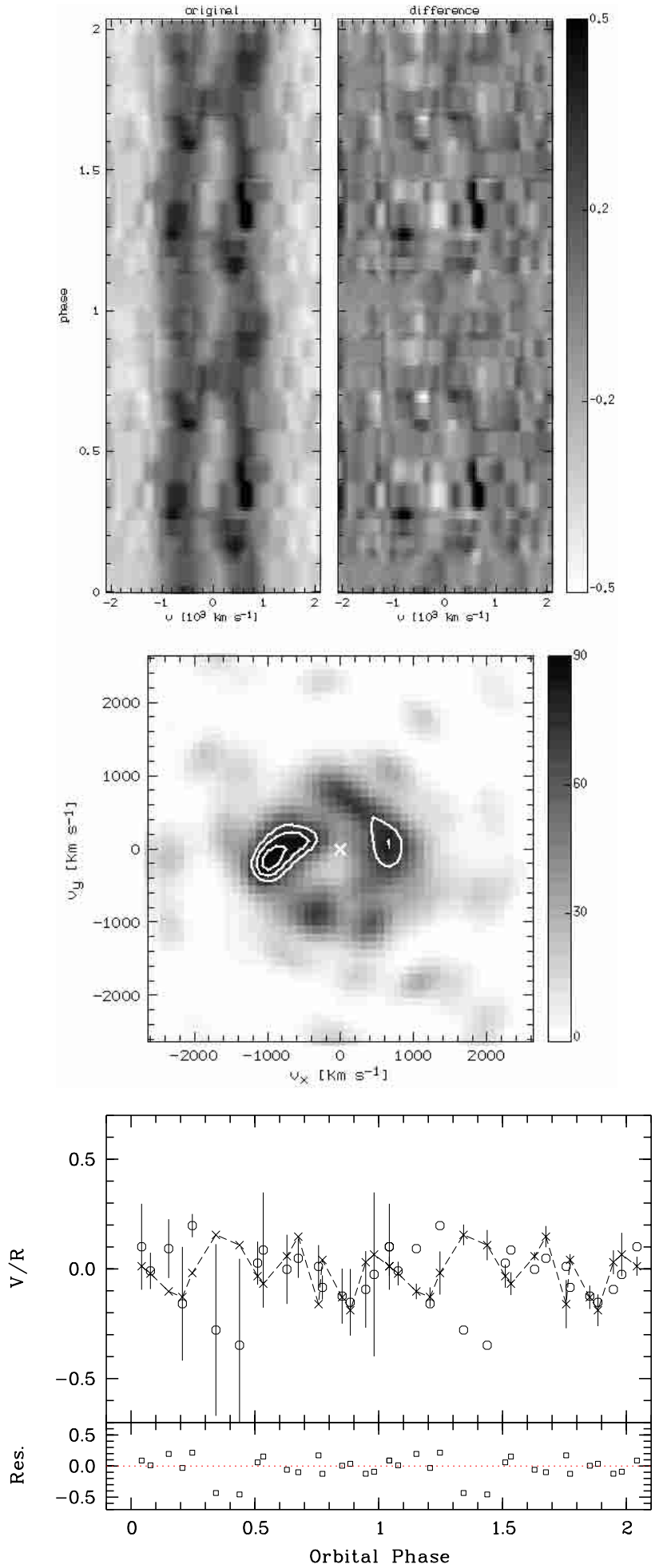

Fig. A.17. Doppler Tomography for RZ Leo, data set 2. Top: original (left) and difference (original-reconstructed; right) spectrum. The intensity bar on the right refers to the latter plot. Middle: doppler map. Contour levels are at 86, 76, and 66 per cent of the maximum intensity. Bottom: $V / R$ plot of the original ( $\circ$ ) and the reconstructed $(\times$ and dashed line) data, and the residuals (bottom of the plot). In phases 0 to 1 , error bars are given for the original data, phases 1 to 2 show those for the reconstructed data.
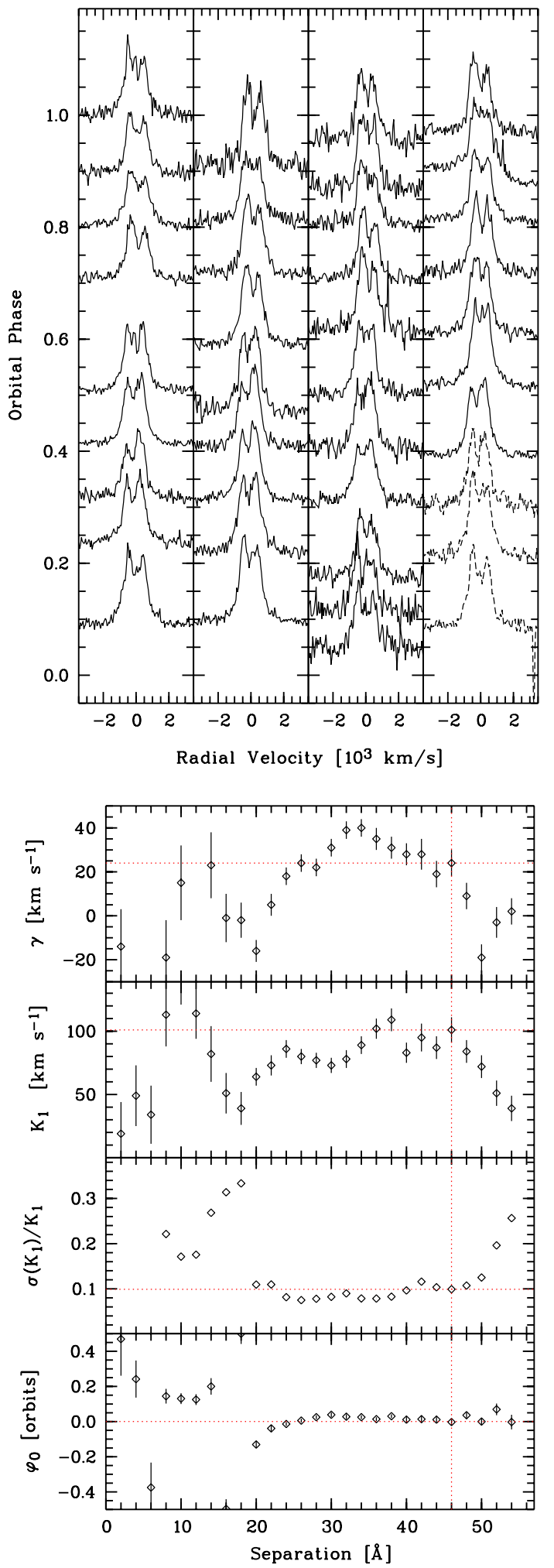

Fig. A.18. Top: line profiles of selected $10 \%$ phase bins for TU Men. Data sets 1 to 4 are plotted from left to right. The time sequence is symbolised by the line styles solid - dashed. Bottom: diagnostic diagram for the combined data set of TU Men, already corrected for the derived zero phase. The dotted lines mark the chosen separation and the corresponding parameters. 
C. Tappert et al.: An atlas of line profile studies for SU UMa stars, Online Material $p 9$
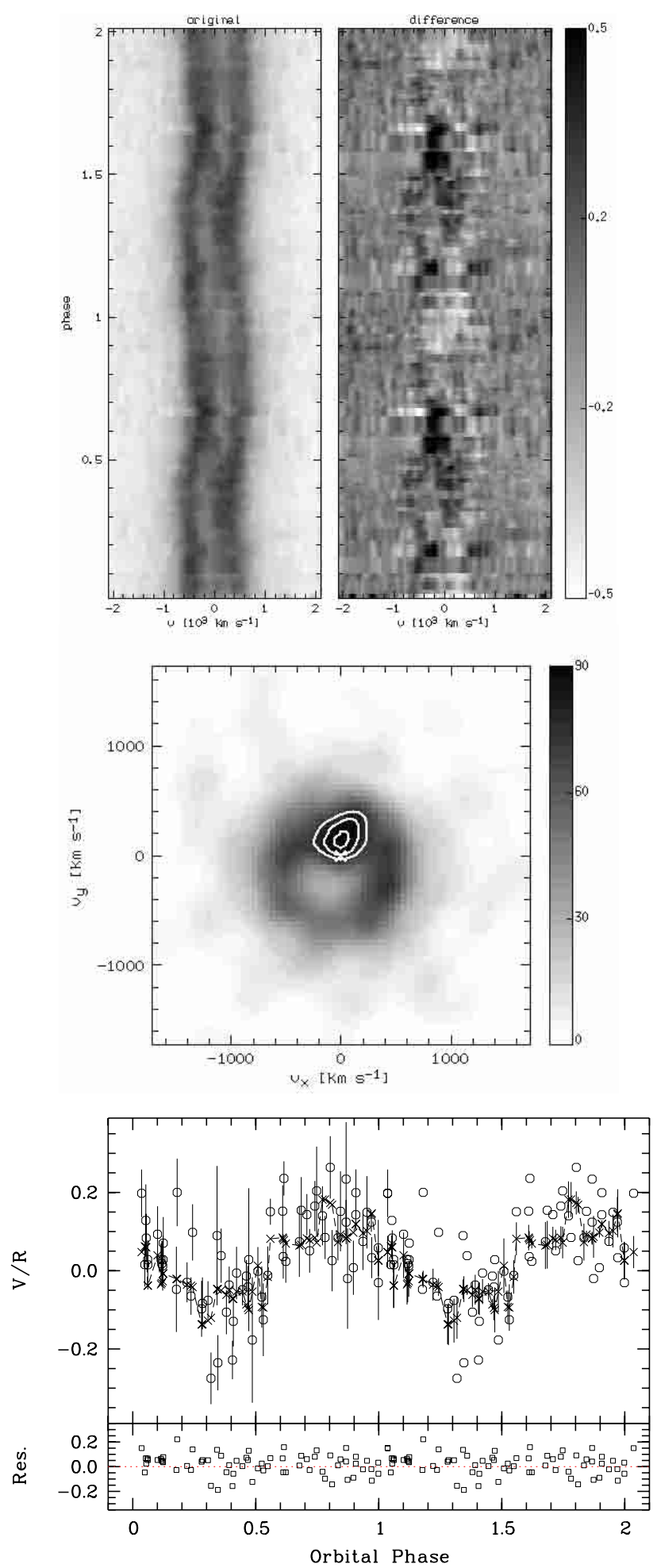

Fig. A.19. Doppler Tomography for TU Men. Top: original (left) and difference (original-reconstructed; right) spectrum. The intensity bar on the right refers to the latter plot. Middle: doppler map. Contour levels are at 94, 84, and 74 per cent of the maximum intensity. Bottom: $V / R$ plot of the original $(\circ)$ and the reconstructed ( $\times$ and dashed line) data, and the residuals (bottom of the plot). In phases 0 to 1 , error bars are given for the original data, phases 1 to 2 show those for the reconstructed data.
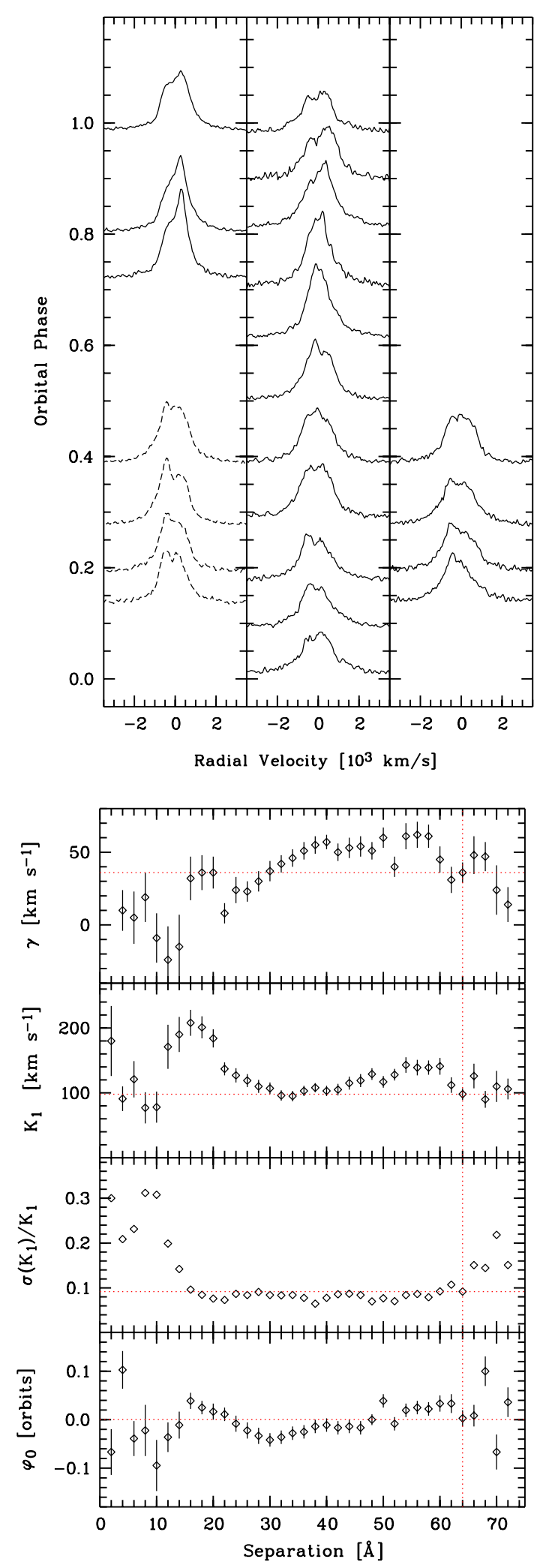

Fig. A.20. Top: line profiles of selected $10 \%$ phase bins for HS Vir. The time sequence in the left plot is symbolised by the sequence solid - dashed. Bottom: diagnostic for HS Vir, already corrected for the derived zero phase. The dotted lines mark the chosen separation and the corresponding parameters. 
C. Tappert et al.: An atlas of line profile studies for SU UMa stars, Online Material p 10
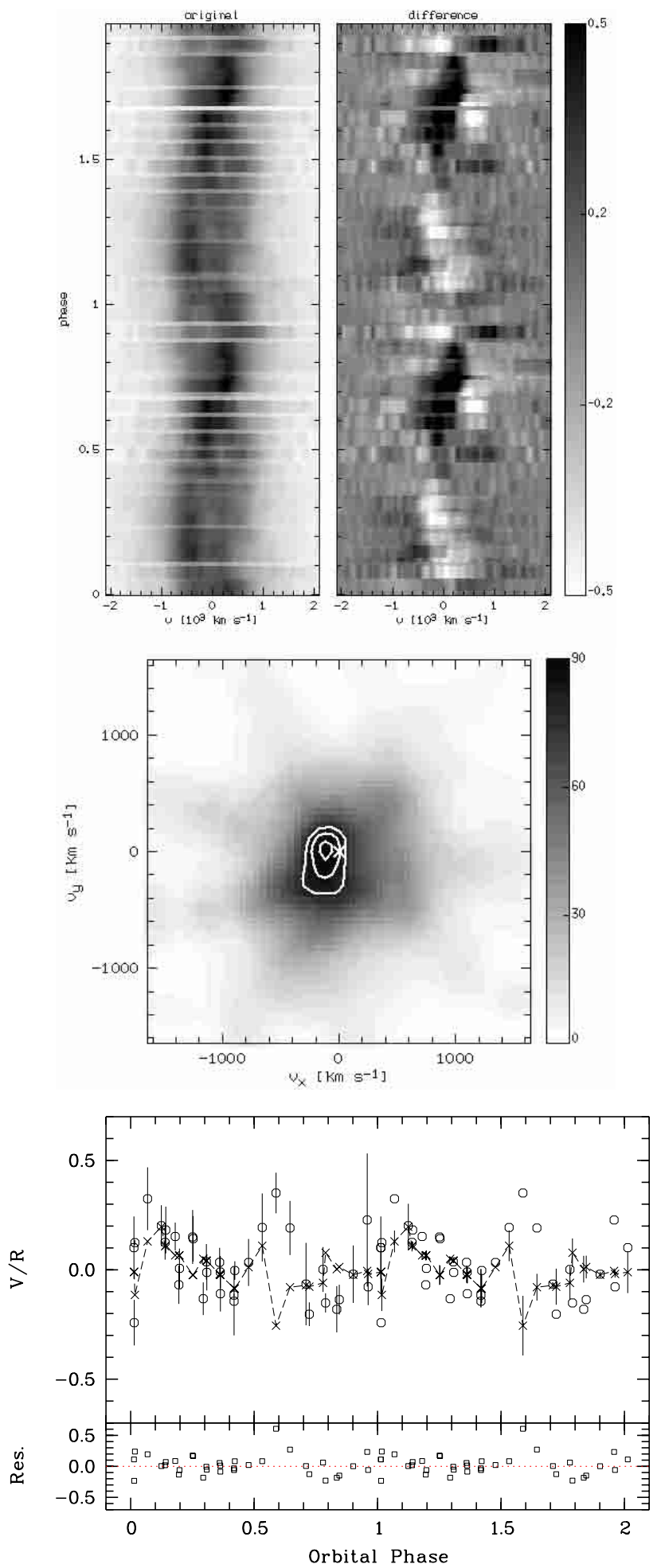

Fig. A.21. Doppler Tomography for HS Vir. Top: original (left) and difference (original-reconstructed; right) spectrum. The intensity bar on the right refers to the latter plot. Middle: doppler map. Contour levels are at 94, 86, and 78 per cent of the maximum intensity. Bottom: $V / R$ plot of the original $(\circ)$ and the reconstructed $(\times$ and dashed line) data, and the residuals (bottom of the plot). In phases 0 to 1 , error bars are given for the original data, phases 1 to 2 show those for the reconstructed data. 